\title{
INFLUENCIA DE LA FUERZA DE INFECCIÓN Y LA TRANSMISIÓN VERTICAL EN LA MALARIA: MODELADO MATEMÁTICO
}

\section{INFLUENCE OF INFECTION FORCE AND VERTICAL TRANSMISSION IN MALARIA: MATHEMATICAL MODELING}

\author{
Jhoana P. ROMERO LEITON ${ }^{1 *}$, Jessica M. MONTOYA ${ }^{2}$, Martín \\ VILLARROEL ${ }^{3}$, Eduardo IBARGÜEN MONDRAGÓN ${ }^{4}$ \\ ${ }^{1}$ Instituto de Matemáticas, Facultad de Ciencias, Universidad de Antioquia \\ ${ }^{2}$ Departamento de Matemáticas, Facultad de Ciencias, Universidad del Quindío \\ ${ }^{3}$ Departamento de Ciencias Básicas, Facultad de Ingeniería, Universidad Minuto de Dios \\ ${ }^{4}$ Departamento de Matemáticas, Facultad de Ciencias Exactas y Naturales, Universidad de \\ Nariño \\ *Autor Corresponsal. E-mail: johana.romeroleiton@udea.edu.co
}

Historia del Artículo

Recibido: Agosto 26, 2016

Evaluado: Diciembre 27, 2016

Aceptado: Enero 16, 2017

Disponible: Febrero 07, 2017

\section{Resumen |}

Dos factores relevantes en la dinámica de transmisión de enfermedades infecciosas son la velocidad con la cual los individuos adquieren la enfermedad, y la transmisión vertical. En epidemiología el primer factor recibe el nombre de fuerza de infección. Este hecho ha motivado el modelado matemático que considere estos factores, sin embargo no siempre se consideran simultáneamente. En este trabajo, se formula y analiza un modelo matemático aplicado a la enfermedad de malaria, en el cual se incorporan ambos factores de forma simultánea. Por otro lado, para la población de mosquitos, además de la fuerza de infección clásica, se introduce una fuerza de infección que depende de la población total de mosquitos.

Palabras Clave: Existencia de soluciones de equilibrio, estabilidad, bifurcaciones, función de Lyapunov.

\section{Abstract}

Two important factors in the transmission dynamics of infectious diseases are the speed at which individuals acquire the disease and vertical transmission. The first factor is called infection force in epidemiology. This has motivated the mathematical modeling that considers these factors, however, they are not always considered simultaneously. In this paper, we formulated and analyzed a mathematical model applied to malaria disease in which both factors are incorporated simultaneously. Furthermore, for the mosquito population, in addition to classical force of infection, we introduce a force of infection that depends on the total mosquito population.

Keywords: Existence of equilibrium solution, stability, bifurcations, Lyapunov function.

\section{INTRODUCCIÓN |}

La malaria es una enfermedad potencialmente mortal con alta incidencia en las regiones tropicales y subtropicales, incluyendo África, Asia, América Latina, Medio Oriente y algunas partes de Europa (The Rollback malaria partnership); esto la convierte en un problema de salud pública de gran relevancia a nivel mundial.

En epidemiología, la fuerza de la infección se define como la tasa per cápita a la cual individuos susceptibles contraen una enfermedad infecciosa (Muench, 1934). Esta tasa depende del número de individuos infecciosos, número de individuos susceptibles y la tasa de contacto entre ambas poblaciones, que a su vez depende de aspectos epidemiológicos y ambientales tales como: densidad poblacional, clima, humedad, precipitación, entre otros. Mueller y sus colaboradores (Mueller et al., 1998), establecen que los factores anteriores contribuyen a la definición de fuerza de infección, la cual puede ser expresada y determinada en términos cuantitativos como sigue

$$
f_{\text {inf }}=\frac{\beta I}{N},
$$

donde $I$ representa el número de individuos capaces de producir infección, $N$ la población total de individuos y $\beta$ es 
el producto entre la tasa de contacto y la probabilidad de transmisión, bajo el supuesto de que el número de contactos es independiente del tamaño de la población. En consecuencia, si $S$ representa la población de individuos suceptibles a contraer la infección entonces $f_{\text {inf }} S$ representa la tasa a la cual se producen nuevas infecciones (Beaumont et al., 2002).

En los modelos matemáticos clásicos de enfermedades transmitidas por vectores se han considerado las siguientes fuerzas de infección de humanos y mosquitos, respectivamente

$$
\beta_{H}=\frac{\beta_{H V} \varepsilon \phi I_{V}}{N_{H}} \mathrm{y} \beta_{1}=\frac{\beta_{V H} \varepsilon \phi I_{H}}{N_{H}},
$$

donde $\beta_{H V}$ representa la probabilidad de infección de un humano por picadura de un mosquito infectado, $\beta_{V H}$ representa la probabilidad de que un mosquito se infecte por picar a un humano infectado, $\varepsilon$ representa la tasa de picadura per cápita de mosquitos y $\phi$ la tasa de inoculación entomológica. En este caso, $\beta_{H} S_{H}$ representa la tasa de aparición de nuevos humanos infectados y $\beta_{V} S_{V}$ la tasa de aparición de nuevos mosquitos infectados. Lo anterior, permite concluir que $\beta_{H}$ y $\beta_{V}$ miden el impacto que produce sobre la población de humanos la introducción un humano infectado (mosquito infectado, respectivamente) en la velocidad de transmisión de la enfermedad. Sin embargo, en ninguna referencia encontrada hasta la fecha, se considera el efecto que produce la introducción de un humano infectado en la población de mosquitos, en la velocidad de transmisión de la malaria, lo cual se representa definiendo la siguiente fuerza de infección para la población de mosquitos

$$
\beta_{2}=\frac{\beta_{V H} \propto \phi I_{H}}{N_{V}},
$$

Por otro lado, en muy pocos casos se ha considerado el impacto de la transmisión vertical en malaria, aspecto que para ciertas regiones es fundamental debido a que la malaria en el embarazo hace parte de la emergencia de salud pública (Fonseca y Maestre, 2009).

El uso de herramientas que describan de manera objetiva y precisa las características de la dinámica de una enfermedad, tales como los modelos matemáticos en ecuaciones diferenciales, permiten predecir su comportamiento y facilitan el desarrollo de estrategias de salud eficaces en el control de la enfermedad y por qué no de su erradicación.

El modelado matemático para la malaria comenzó en 1902 con Sir Ronald Ross (Ross, 1911), quien fue galardonado con el premio Nobel en Medicina por descubrir que esta enfermedad se transmite a través de la picadura de la hembra del mosquito Anopheles. Con su modelo Ross mostró a sus colegas que para erradicar la malaria no es necesario eliminar a los mosquitos, sino que es suficiente mantener su nivel poblacional por debajo de un umbral. En 1927, Kermack y McKendrick (Sobrebon et al., 2002) formulan un modelo matemático en ecuaciones integrodiferenciales con retardo que modela el flujo de susceptibles, infectados $y$ recuperados en un proceso epidémico. Usando una versión simplificada de este modelo obtienen el llamado Teorema del Umbral, piedra angular de la Epidemiología Matemática. Este resultado establece condiciones sobre las tasas de infección y recuperación, así como en el número inicial de susceptibles para que la introducción de un individuo infeccioso en una comunidad, de lugar a un brote epidémico. En 1957, MacDonald (Macdonald, 1957) modificó el modelo de Kermack y McKendrick a un modelo de dos dimensiones con una variable para representar la población de humanos y otra para representar los mosquitos. Una importante extensión de este modelo fue propuesta por Dietz, Molineaux y Thomas (Dietz, 1974), quienes agregaron la inclusión de la inmunidad. Ngwa and Shu (Nwa y Shu, 2000), propusieron un modelo en ecuaciones diferenciales ordinarias en el cual incluyeron cuatro diferentes estados para los humanos (susceptible-expuesto-infectadorecuperado) y tres diferentes estados para los mosquitos (susceptible-expuesto-infectado), donde estos estados interactúan a tasas diferentes. El modelo de Ngwa y Shu fue después estudiado y mejorado por Chitnis (Chitnis et al., 2006; Chitnis et al., 2008).

Hasta el momento se han formulado modelos matemáticos con fragmentación espacial del ambiente (Torres-Sorando et al., 1997; Rodriguez et al., 2001), modelos para enfermedades transmitidas por vectores en los cuales se considera resistencia a medicamentos (Esteva y Yang, 2005; Tchuenche et al., 2011), modelos en los cuales se supone que los humanos infectados poseen una movilidad inferior que los humanos susceptibles (Basañez, 2004), y también modelos para la distribución espacial de la malaria (Rainey et al., 2007). Los modelos multiparche han sido construidos para estudiar la propagacíón espacial de las enfermedades infecciosas cuando hay viaje de poblaciones de una región a otra (Hasibeder y Dye, 1988; Dye y Hasibeder, 1986 ; Smith et al., 2004; Auger et al., 2008; Arino et al., 2012; Gao y Ruan, 2012; Crosner et al., 2009).

En el presente trabajo se modela usando un sistema de ecuaciones diferenciales ordinarias, el fenómeno de transmisión de la malaria cuando esta enfermedad no solamente se adquiere por transmisión vectorial, sino también vertical, con el fin de comparar el impacto de los resultados del modelo con la fuerza de infección de los humanos contra la fuerza de infección de los mosquitos.

\section{MODELO MATEMÁTICO Y CONJUNTO DE} INTERÉS EPIDEMIOLÓGICO |

En esta sección se formula un sistema de ecuaciones diferenciales ordinarias que describe la interacción de humanos y mosquitos en la epidemiología de la malaria bajo 
las siguientes hipótesis: para la población humana el modelo matemático es de tipo Susceptible-Expuesto-InfectadoRecuperado (SEIR), mientras que para la población de mosquitos se utiliza un modelo matemático tipo SusceptibleInfectado (SI). El corto ciclo de vida de los mosquitos, particularmente reducido por los constantes cambios de clima, hace que sea irrelevante considerar el paso por el estadío de expuestos, mientras que para la población humana considerar su periodo de latencia, hace que no sólo se reduzca a largo plazo la población de individuos infectados sino que también las tasas de progresión a expuestos e infectados (Mandal et al., 2011). $S_{H}(t), E_{H}(t)$, $I_{H}(t)$ y $R_{H}(t)$ denotan, respectivamente, la población de humanos susceptibles, expuestos, infectados y recuperados en el tiempo $t$, y $S_{V}(t), I_{V}(t)$ las poblaciones de mosquitos susceptibles e infectados en un tiempo $t$, respectivamente. Por conveniencia se redefine la fuerza de infección de humanos dada en (2) como

$$
\beta_{H}=\frac{\beta_{h}}{N_{H}} I_{V},
$$

donde $\beta_{h}=\beta_{H V} \partial \phi$, análogamente se redefinen las fuerzas de infección de mosquitos dadas en (2) y (3) por

$$
\beta_{1}=\frac{\beta_{v}}{N_{H}} I_{H} \quad \text { y } \quad \beta_{2}=\frac{\beta_{v}}{N_{V}} I_{H},
$$

donde $\beta_{v}=\beta_{V H} \not \phi$. En el modelo se considera que los humanos susceptibles se reclutan a una tasa constante $\Lambda_{H}$, pasan a ser expuestos por contacto con mosquitos infectados a una tasa $\beta_{H} S_{H}$. Además presentan una muerte natural o emigración percápita $\mu_{H} S_{H}$. Los humanos expuestos se infectan a una tasa percápita $\alpha S_{H}$ donde $\alpha$ representa el periodo de incubación de la enfermedad. Otro factor que incrementa la población de humanos infectados es la transmisión vertical, el cual es modelado por el término $\lambda I_{H}$, donde $\lambda$ representa la tasa percápita de transmisión vertical. Por otro lado, los humanos infectados se recuperan a una tasa $\delta I_{H}$, donde $\delta$ representa la tasa de recuperación espontánea y mueren debido a la infección a una tasa percápita $\rho I_{H}$. Una proporción de humanos recuperados de la infección pasan a ser susceptibles nuevamente a una tasa percápita $\omega R_{H}$, donde $\omega$ representa una tasa percápita de pérdida de inmunidad temporal. La población de mosquitos susceptibles se recluta a una tasa constante $\Lambda_{V}$, se ve disminuida por infección debido al contacto con humanos infectados mediante el término $\beta_{i} S_{V}$ para $i=1,2$. La población de mosquitos susceptibles e infectados disminuyen por muerte o emigración percápita con constante de mortalidad o emigración $\mu_{v}$.

Bajo las consideraciones anteriores, se formula el siguiente sistema de ecuaciones diferenciales ordinarias,

$$
\frac{d S_{H}}{d t}=\Lambda_{H}+\omega R_{H}-\beta_{H} S_{H}-\mu_{H} S_{H}
$$

$$
\begin{aligned}
\frac{d E_{H}}{d t} & =\beta_{H} S_{H}-\left(\alpha+\mu_{H}\right) E_{H} \\
\frac{d I_{H}}{d t} & =\lambda I_{H}+\alpha E_{H}-\left(\delta+\rho+\mu_{H}\right) I_{H} \\
\frac{d R_{H}}{d t} & =\delta I_{H}-\left(\omega+\mu_{H}\right) R_{H} \\
\frac{d S_{V}}{d t} & =\Lambda_{V}-\beta_{i} S_{V}-\mu_{V} S_{V} \\
\frac{d I_{V}}{d t} & =\beta_{i} S_{V}-\mu_{V} I_{V} \quad \text { para } \quad i=1,2 .
\end{aligned}
$$

\begin{tabular}{|c|c|c|}
\hline Parámetro & Dimensión & Interpretación \\
\hline$\Lambda_{H}$ & Hum. X día ${ }^{-1}$ & Tasa de reclutamiento o inmigración de hum. \\
\hline$\omega$ & $d^{d} a^{-1}$ & $\begin{array}{l}\text { Tasa per. de pérdida de inmuni. } 1 / \omega \text { prom. } \\
\text { duración período inmune }\end{array}$ \\
\hline$\beta_{H V}$ & Adimensional & Prob. de infección de hum. por mosq. \\
\hline$\beta_{V H}$ & Adimensional & Prob. de infección de mosq. por hum. \\
\hline$\varepsilon$ & día $^{-1}$ & Tasa de picadura percápita de mosq. \\
\hline$\phi$ & día $^{-1}$ & Tasa percápita de inoculación entomológica \\
\hline$\mu_{H}$ & día $^{-1}$ & $\begin{array}{l}\text { Tasa percápita muerte natural o emigración de } \\
\text { hum. }\end{array}$ \\
\hline$\mu_{V}$ & día $^{-1}$ & $\begin{array}{l}\text { Tasa percápita muerte natural o emigración de } \\
\text { mosq. }\end{array}$ \\
\hline$\alpha$ & día $^{-1}$ & $\begin{array}{l}\text { Tasa per. de progresión de expue. a infect. } \\
1 / \alpha \text { duración de período de laten. }\end{array}$ \\
\hline$\rho$ & día $^{-1}$ & Tasa percápita de muerte por infección \\
\hline$\delta$ & día $^{-1}$ & $\begin{array}{l}\text { Tasa per. de recuperación. } 1 / \delta \text { duración del } \\
\text { período infeccioso }\end{array}$ \\
\hline$\lambda$ & día $^{-1}$ & Tasa percápita de transmisión vertical \\
\hline$\Lambda_{V}$ & Mos. X día & $\begin{array}{l}\text { Tasa de reclutamiento o inmigración de } \\
\text { mosquitos }\end{array}$ \\
\hline
\end{tabular}

La Tabla 1 muestra los parámetros incluidos en el modelo matemático anterior, con sus unidades y respectiva interpretación, en donde la unididad de tiempo es un día.

\subsection{Conjunto de interés epidemiológico}

El conjunto de interés biológico para el sistema (6) está dado por

$\Omega=\left\{\left(S_{H}, E_{H}, I_{H}, R_{H}, S_{V}, I_{V}\right) \in \mathrm{R}_{+}^{6}: 0 \leq N_{H} \leq m, \quad 0 \leq N_{V} \leq \frac{\Lambda_{V}}{\mu_{V}}\right\}$,

donde

$$
\rho^{*}=\frac{\lambda-\rho}{\mu_{H}}, \quad m=\min \left(\frac{\Lambda_{H}}{\mu_{H}}, \frac{\Lambda_{H}}{\mu_{H}\left(1-\rho^{*}\right)}\right) .
$$

Obsérvese que $\rho^{*}<1$ es una condición necesaria para que $m$ siempre sea positiva. En el Lema 2.1 se prueba que el sistema (6) está bien planteado en el sentido de que las soluciones con condiciones iniciales no negativas se mantienen no negativas para todo $t \geq 0$, es decir, el conjunto $\Omega$ es positivamente invariante bajo el flujo definido por el sistema de ecuaciones diferenciales (6).

Lema 2.1 Si $\rho^{*}<1$, entonces el conjunto $\Omega$ definido en (7) es positivamente invariante para las soluciones del sistema (6).

Demostración. Considérense las condiciones iniciales $N_{H}^{0} \times N_{V}^{0} \in \Omega$ donde 
$N_{H}^{0}=\left(S_{H}(0), E_{H}(0), I_{H}(0), R_{H}(0)\right), \quad N_{V}^{0}=\left(S_{V}(0), I_{V}(0)\right)$

Sumando las cuatro primeras ecuaciones del sistema (6) se obtiene que

$$
\frac{d N_{H}}{d t}=\Lambda_{H}-\mu_{H} N_{H}+\mu_{H} \rho^{*} I_{H} .
$$

Si $\rho^{*} \leq 0$ entonces a partir de (9) se obtiene la siguiente desigualdad

$$
\dot{N}_{H} \leq \Lambda_{H}-\mu_{H} N_{H},
$$

la cual se reescribe como

$$
\frac{N_{H}}{d t}+\mu_{H} N_{H} \leq \Lambda_{H}
$$

Obsérvese que la desigualdad (10) es equivalente a

$$
\frac{d}{d t}\left[e^{\mu_{H} t} N_{H}\right] \leq \Lambda_{H} e^{\mu_{H} t},
$$

integrando en ambos lados de (11) entre 0 y $t$ se obtiene

$$
\begin{aligned}
\left.e^{\mu_{H} t} N_{H}(s)\right|_{0} ^{t} & \leq\left.\frac{\Lambda_{H}}{\mu_{H}} e^{\mu_{H} s}\right|_{0} ^{t} \\
e^{\mu_{H} t} N_{H}(t)-N_{H}(0) & \leq \frac{\Lambda_{H}}{\mu_{H}}\left(e^{-\mu_{H} t}-1\right),
\end{aligned}
$$

a partir de (12) y con la condición inicial se obtiene

$$
\begin{aligned}
N_{H}(t) & \leq N_{H}^{0} e^{-\mu_{H} t}+\frac{\Lambda_{H}}{\mu_{H}}\left(1-e^{-\mu_{H} t}\right) \\
& =\frac{\Lambda_{H}}{\mu_{H}}+\left(N_{H}^{0}-\frac{\Lambda_{H}}{\mu_{H}} e^{-\mu_{H} t}\right),
\end{aligned}
$$

dado que $N_{H}^{0} \in \Omega$, entonces $N_{H}^{0} \leq \frac{\Lambda_{H}}{\mu_{H}}$, luego

$$
\begin{aligned}
N_{H}^{0}-\frac{\Lambda_{H}}{\mu_{H}} & \leq 0 \\
\frac{\Lambda_{H}}{\mu_{H}}+\left(N_{H}^{0}-\frac{\Lambda_{H}}{\mu_{H}}\right) e^{-\mu_{H} t} & \leq \frac{\Lambda_{H}}{\mu_{H}} \\
N_{H}(t) & \leq \frac{\Lambda_{H}}{\mu_{H}} .
\end{aligned}
$$

Por otro lado, si $0<\rho^{*}<1$ y dado que $I_{H}(t) \leq N_{H}(t)$, entonces a partir de (9) se tiene

$$
\begin{aligned}
\dot{N}_{H} & \leq \Lambda_{H}+\mu_{H} \rho^{*} N_{H}-\mu_{H} N_{H} \\
& =\Lambda_{H}+\left(\rho^{*}-1\right) \mu_{H} N_{H},
\end{aligned}
$$

la solución de (15) sujeta a la condición inicial es

$$
N_{H}(t) \leq N_{H}^{0} e^{-\mu_{H}^{\left(1-\rho^{*}\right) t}}+\frac{\Lambda_{H}}{\mu_{H}\left(1-\rho^{*}\right)}\left(1-e^{-\mu_{H}\left(1-\rho^{*}\right) t}\right)
$$

$$
=\frac{\Lambda_{H}}{\mu_{H}\left(1-\rho^{*}\right)}+\left(N_{H}^{0}-\frac{\Lambda_{H}}{\mu_{H}\left(1-\rho^{*}\right)} e^{-\mu_{H}^{\left(1-\rho^{*}\right) t}}\right)
$$

entonces $N_{H}^{0} \leq \frac{\Lambda_{H}}{\mu_{H}\left(1-\rho^{*}\right)}$, luego

$$
N_{H}^{0}-\frac{\Lambda_{H}}{\mu_{H}\left(1-\rho^{*}\right)} \leq 0
$$

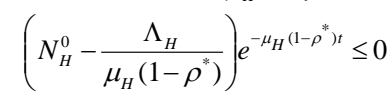

$\frac{\Lambda_{H}}{\mu_{H}\left(1-\rho^{*}\right)}+\left(N_{H}^{0}-\frac{\Lambda_{H}}{\mu_{H}\left(1-\rho^{*}\right)}\right) e^{-\mu_{H}\left(1-\rho^{*}\right) t} \leq \frac{\Lambda_{H}}{\mu_{H}\left(1-\rho^{*}\right)}$

$$
N_{H}(t) \leq \frac{\Lambda_{H}}{\mu_{H}\left(1-\rho^{*}\right)},
$$

luego por (14), (17) y con base a la Definición de $m$ dada en (8) se concluye que

$$
N_{H}(t) \leq m \text {. }
$$

De manera análoga tomando las dos últimas ecuaciones de (6) se tiene que

$$
\dot{N}_{V}=\Lambda_{V}-\mu_{V} N_{V}
$$

la cual puede reescribirse de la siguiente manera

$$
\frac{d N_{V}}{d t}+\mu_{V} N_{V}=\Lambda_{V}
$$

solucionando la ecuación (19) se obtiene

$$
\begin{aligned}
N_{V}(t) & =e^{-\mu_{V} t} N_{V}^{0}+\frac{\Lambda_{V}}{\mu_{V}}\left(1-e^{-\mu_{V} t}\right) \\
& =\frac{\Lambda_{V}}{\mu_{V}}+\left(N_{V}^{0}-\frac{\Lambda_{V}}{\mu_{V}}\right) e^{-\mu_{V} t},
\end{aligned}
$$

dado que $N_{V}^{0} \in \Omega$, entonces $N_{V}^{0} \leq \frac{\Lambda_{V}}{\mu_{V}}$, luego

$$
\begin{aligned}
N_{V}^{0}-\frac{\Lambda_{V}}{\mu_{V}} & \leq 0 \\
\frac{\Lambda_{V}}{\mu_{V}}+\left(N_{V}^{0}-\frac{\Lambda_{V}}{\mu_{V}}\right) e^{-\mu_{V} t} & \leq \frac{\Lambda_{V}}{\mu_{V}} \\
N_{V}(t) & \leq \frac{\Lambda_{V}}{\mu_{V}} .
\end{aligned}
$$

Finalmente, evaluando el campo vectorial definido en la parte derecha del sistema (6) en la frontera de $\Omega$, se verifica que el vector director de dicho campo apunta hacia el interior de $\Omega$. De lo anterior y puesto que el campo vectorial definido en la parte derecha de (6) es continuamente diferenciable se sigue el resultado así como también existencia y unicidad de soluciones.

\section{SOLUCIONES DE EQUILIBRIO}

En esta sección se determinan la soluciones de equilibrio del sistema (6), en particular se verifica que la 
solución de equilibrio libre de infección denotada como $E_{0}$ es única sin importar la fuerza de infección de los mosquitos considerada. Obsérvese que las soluciones de equilibrio de (6) se obtienen solucionando el siguiente sistema de ecuaciones algebraicas

$$
\begin{aligned}
\Lambda_{H}+\omega R_{H}-\left(\beta_{H}+\mu_{H}\right) S_{H}=0, & \beta_{H} S_{H}-\left(\alpha+\mu_{H}\right) E_{H}=0 \\
\alpha E_{H}-\left(\delta+\rho+\mu_{H}-\lambda\right) I_{H}=0, & \delta I_{H}-\left(\omega+\mu_{H}\right) R_{H}=0 \\
\Lambda_{V}-\left(\beta_{i}+\mu_{V}\right) S_{V}=0, & \beta_{i} S_{V}-\mu_{V} I_{V}=0 \quad i=1,2 .
\end{aligned}
$$

Cuando $E_{H}=I_{H}=R_{H}=I_{V}=0$ el sistema (22) tiene una única solución de equilibrio libre de infección $E_{0}$ dada por

$$
E_{0}=\left(\frac{\Lambda_{H}}{\mu_{H}}, 0,0,0, \frac{\Lambda_{V}}{\mu_{V}}, 0\right) .
$$

Ahora, se analizarán las soluciones de equilibrio endémicas de (6) cuando alguna de las fuerzas de infección es constante o cuando ninguna lo es. Para este fin, se definen los siguientes parámetros

$$
\rho_{1}=\frac{\beta_{v}}{\mu_{V}}>0 \quad \text { y } \quad 0<\theta=\frac{\omega}{\omega+\mu_{H}} \frac{\alpha}{\alpha+\mu_{H}} \frac{\delta}{\mu_{H}\left(1-\rho^{*}\right)+\delta} \leq 1 .
$$

3.1. Caso 1: alguna de las fuerzas de infección es constante

Se inicia considerando $\beta_{1}$ constante. Despejando $I_{H}$ de (5) se obtiene

$$
I_{H}=\frac{\beta_{1}}{\beta_{v}} N_{H} .
$$

Sumando las cuatro primeras ecuaciones de (22) se obtiene la siguiente ecuación

$$
\Lambda_{H}-\mu_{H} N_{H}-\left(\rho-\lambda_{H}\right) I_{H}=0 .
$$

Reemplazando (25) en (26), y despejando $N_{H}$ se obtiene

$$
N_{H}=\frac{\Lambda_{H}}{1-\rho^{*} \frac{\beta_{1}}{\beta_{v}}} .
$$

Obsérvese que $N_{H}$ definido en (27) es positivo si y sólo si se satisface que

$$
\rho^{*}<\frac{\beta_{v}}{\beta_{1}} .
$$

Ahora, reemplazando (27) en (25) se obtiene

$$
I_{H}=\frac{\Lambda_{H}}{\frac{\beta_{v}}{\beta_{1}}-\rho^{*}} .
$$

Reemplazando (29) en la tercera y cuarta ecuación de (22) y despejando $E_{H}$ y $R_{H}$ respectivamente se obtiene

$$
E_{H}=\frac{\mu_{H}\left(1-\rho^{*}\right)+\delta}{\alpha} \frac{\Lambda_{H}}{\frac{\beta_{v}}{\beta_{1}}-\rho^{*}}, R_{H}=\frac{\delta}{\omega+\mu_{H}} \frac{\Lambda_{H}}{\frac{\beta_{v}}{\beta_{1}}-\rho^{*}} .
$$

En seguida, sumando las dos primeras ecuaciones de (22) y despejando $S_{H}$ se obtiene

$$
S_{H}=\frac{\Lambda_{H}+\omega R_{H}-\left(\alpha+\mu_{H}\right) E_{H}}{\mu_{H}} .
$$

Reemplazando (30) en (31) se obtiene

$$
S_{H}=\frac{\Lambda_{H}}{\mu_{H}}\left(1-\frac{1}{R_{1}}\right),
$$

donde

$$
R_{1}=\frac{\alpha\left(\frac{\beta_{v}}{\beta_{1}}-\rho^{*}\right)}{(1-\theta)\left(\alpha+\mu_{H}\right)\left(\mu_{H}\left(1-\rho^{*}\right)+\delta\right)} .
$$

A partir de la quinta y sexta ecuación de (22) se obtiene

$$
S_{V}=\frac{\Lambda_{V}}{\beta_{1}+\mu_{V}} \quad \text { y } \quad I_{V}=\frac{\beta_{1}}{\mu_{V}} \frac{\Lambda_{V}}{\beta_{1}+\mu_{V}} .
$$

Los resultados de existencia de soluciones de equilibrio para la fuerza de infección $\beta_{1}$ constante se resumen en la Proposición 3.1.

Proposición 3.1 El sistema (6) siempre tiene un equilibrio libre de infección $E_{0}$ definido en (23). Además, cuando $\beta_{1}$ es constante se tiene que

1. $S i^{\rho^{*}<\frac{\beta_{v}}{\beta_{1}}}$ entonces

(a) Si $R_{1} \leq 1$ no existen equilibrios endémicos.

(b) $\mathrm{Si} R_{1}>1$ existe un equilibrio endémico.

2. $S i \rho^{*} \geq \frac{\beta_{v}}{\beta_{1}}$ no existen equilibrios endémicos.

Para $\beta_{2}$ y $\beta_{H}$ constantes, siguiendo un procedimiento similar al anterior se obtienen los resultados dados en las Proposiciones 3.2 y 3.3, respectivamente.

Proposición 3.2 Sea

$$
R_{2}=\frac{\lambda}{\mu_{H}} \frac{\mu_{V}}{\Lambda_{V}} \frac{\alpha \mu_{H}}{(1-\theta)\left(\alpha+\mu_{H}\right)\left(\mu_{H}\left(1-\rho^{*}\right)+\delta\right)} \frac{\beta_{v}}{\beta_{2}} .
$$

El sistema (6) siempre tiene un equilibrio libre de infección $E_{0}$ definido en (23). Además, si $\beta_{2}$ es constante se tiene que

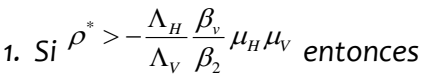

(a) Si $R_{2} \leq 1$ no existen equilibrios endémicos.

(b) Si $R_{2}>1$ existe un equilibrio endémico.

2. $\mathrm{Si} \rho^{*} \leq-\frac{\Lambda_{H}}{\Lambda_{V}} \frac{\beta_{v}}{\beta_{2}} \mu_{H} \mu_{V}$ no existen equilibrios endémicos.

Proposición 3.3 Sea 


$$
R_{3}=\frac{\Lambda_{V}}{\mu_{V}} \frac{\beta_{v}}{\beta_{H}} \frac{1}{\tilde{N}_{H}},
$$

con $\tilde{N}_{H}$ un parámetro positivo. Entonces el sistema (6) siempre tiene un equilibrio libre de infección $E_{0}$ definido en (23). Además, cuando $\beta_{H}$ es constante se tiene que

1. Si $R_{3} \leq 1$ no existen equilibrios endémicos.

2. Si $R_{3}>1$ existe un equilibrio endémico.

Nota 3.1 La razón epidemiológica de considerar las fuerzas de infección para humanos y mosquitos constantes, es el hecho de que en la naturaleza existen poblaciones en las cuales el cociente entre el número total de individuos y el número total de individuos infectados es constante en el tiempo. Bajo esta hipótesis los resultados obtenidos en las Proposiciones 3.1-3.3 indican que con el fin de controlar la infección, basta tomar medidas que garanticen mantener el umbral $R_{j}, j=1,2,3$ por debajo de uno.

\subsection{Caso 2: ninguna fuerza de infección es constante}

Se inicia el análisis considerando la fuerza de infección de mosquitos $\beta_{1}$ definida en (5). En este caso se define el número reproductivo básico $R_{0}$ como

$$
R_{0}=\sqrt{\beta_{h} \frac{\Lambda_{V} / \mu_{V}}{\Lambda_{H} / \mu_{H}} \frac{\alpha}{\alpha+\mu_{H}} \frac{1}{\mu_{H}\left(1-\rho^{*}\right)+\delta} \beta_{v} \frac{1}{\mu_{V}}},
$$

el cual será calculado en la Sección 4.1. Sumando las cuatro primeras ecuaciones de (22) se obtiene que

$$
\Lambda_{H}-\mu_{H} N_{H}+(\lambda-\rho) I_{H}=0,
$$

de donde

$$
N_{H}=\frac{\Lambda_{H}}{\mu_{H}}+\rho^{*} I_{H} .
$$

Análogamente, sumando las dos últimas ecuaciones de (22) se obtiene

$$
\Lambda_{V}-\mu_{V}\left(S_{V}+I_{V}\right)=0
$$

de donde

$$
S_{V}=\frac{\Lambda_{V}}{\mu_{V}}-I_{V} .
$$

A partir de la tercera y cuarta ecuación de (22) se obtiene

$$
E_{H}=\frac{\mu_{H}\left(1-\rho^{*}\right)+\delta}{\alpha} I_{H}, R_{H}=\frac{\delta}{\omega+\mu_{H}} I_{H} .
$$

Sumando las dos primeras ecuaciones de (22) se obtiene

$$
\Lambda_{H}+\omega R_{H}-\left(\alpha+\mu_{H}\right) E_{H}-\mu_{H} S_{H}=0 .
$$

Despejando $S_{H}$ de la anterior ecuación

$$
S_{H}=\frac{\Lambda_{H}+\omega R_{H}-\left(\alpha+\mu_{H}\right) E_{H}}{\mu_{H}} .
$$

Reemplazando $R_{H}$ y $E_{H}$ definidos en (39) en (40) se sigue que

$$
S_{H}=\frac{\Lambda_{H}}{\mu_{H}}-\frac{\left(\alpha+\mu_{H}\right)\left(\mu_{H}\left(1-\rho^{*}\right)+\delta\right)}{\alpha \mu_{H}}(1-\theta) I_{H} .
$$

Reemplazando $\beta_{1}$ definido en (5) en la sexta ecuación de (22) se obtiene

$$
\frac{\beta_{v} I_{H} S_{V}}{N_{H}}=\mu_{V} I_{V} .
$$

Reemplazando $S_{V}$ definido en (38) y $N_{H}$ definido en (37) en (42) se obtiene

$$
\beta_{v} I_{H}\left(\frac{\Lambda_{V}}{\mu_{V}}-I_{V}\right)=\mu_{V} I_{V}\left(\frac{\Lambda_{H}}{\mu_{H}}+\rho^{*} I_{H}\right) .
$$

Despejando $I_{V}$ de (43)

$$
I_{V}=\frac{\Lambda_{V}}{\mu_{V}} \frac{\beta_{v} I_{H}}{\mu_{V} \frac{\Lambda_{H}}{\mu_{H}}+\left(\beta_{v}+\mu_{V} \rho^{*}\right) I_{H}} .
$$

Reemplazando (44) en (38) se obtiene

$$
S_{V}=\frac{\Lambda_{V}}{\mu_{V}}\left(1-\frac{\beta_{v} I_{H}}{\mu_{V} \frac{\Lambda_{H}}{\mu_{H}}+\left(\beta_{v}+\mu_{V} \rho^{*}\right) I_{H}}\right) .
$$

Reemplazando $\beta_{H}$ definido en (4), $S_{H}$ definido en (41) y $E_{H}$ definido en (39) en la segunda ecuación de (22) se obtiene

$$
\begin{gathered}
\frac{\beta_{h} I_{V}}{N_{H}}\left(\frac{\Lambda_{H}}{\mu_{H}}-\frac{\left(\alpha+\mu_{H}\right)\left(\mu_{H}\left(1-\rho^{*}\right)+\delta\right)}{\alpha \mu_{H}}(1-\theta) I_{H}\right) \\
=\frac{\left(\alpha+\mu_{H}\right)\left(\mu_{H}\left(1-\rho^{*}\right)+\delta\right)}{\alpha} I_{H} .
\end{gathered}
$$

La expresión anterior es equivalente a

$$
\beta_{h} I_{V}\left(\frac{\Lambda_{H}}{\mu_{H}} \frac{\alpha}{\left(\alpha+\mu_{H}\right)\left(\mu_{H}\left(1-\rho^{*}\right)+\delta\right)}-\frac{1}{\mu_{H}}(1-\theta) I_{H}\right)=I_{H} N_{H} .
$$

Reemplazando $I_{V}$ definido en (44) y $N_{H}$ definido en (37) en la anterior ecuación se obtiene

$$
\begin{gathered}
\frac{\Lambda_{V}}{\mu_{V}} \frac{\beta_{h} \beta_{v} I_{H}}{\mu_{V} \frac{\Lambda_{H}}{\mu_{H}}+\left(\beta_{v}+\mu_{V} \rho^{*}\right) I_{H}}\left(\frac{\Lambda_{H}}{\mu_{H}} \frac{\alpha}{\left(\alpha+\mu_{H}\right)\left(\mu_{H}\left(1-\rho^{*}\right)+\delta\right)}-\frac{1}{\mu_{H}}(1-\theta) I_{H}\right) \\
=I_{H}\left(\frac{\Lambda_{H}}{\mu_{H}}+\rho^{*} I_{H}\right) .
\end{gathered}
$$

Como $I_{H} \neq 0$, pues se está considerando existencia de soluciones de equilibrio endémicas, usando (24) y (36), la ecuación anterior puede simplificarse como la siguiente ecuación cuadrática para $I_{H}$ 


$$
\rho^{*}\left(\rho_{1}+\rho^{*}\right) I_{H}^{2}+\frac{\Lambda_{H}}{\mu_{H}}\left[\rho_{1}+2 \rho^{*}+\frac{(1-\theta) \beta_{h} \Lambda_{V} \rho_{1}}{\Lambda_{H} \mu_{V}}\right] I_{H}+\left(\frac{\Lambda_{H}}{\mu_{H}}\right)^{2}\left(1-R_{0}^{2}\right)=0 .
$$

Simplificando los coeficientes la ecuación anterior, se reescribirse como

$$
a\left(I_{H}\right)^{2}+b I_{H}+c=0,
$$

donde

$$
a=\rho^{*}\left(\rho_{1}+\rho^{*}\right), b=A\left(R_{0}^{2}-L\right), c=B\left(1-R_{0}^{2}\right),
$$

siendo

$$
A=\frac{\Lambda_{H}\left(\alpha+\mu_{H}\right)\left(\mu_{H}\left(1-\rho^{*}\right)+\delta\right)(1-\theta)}{\alpha \mu_{H}^{2}}, B=\left(\frac{\Lambda_{H}}{\mu_{H}}\right)^{2}>0,
$$

y además

$$
L=-\frac{\alpha \mu_{H}}{\left(\alpha+\mu_{H}\right)\left(\mu_{H}\left(1-\rho^{*}\right)+\delta\right)(1-\theta)}\left(\rho_{1}+2 \rho^{*}\right) .
$$

En el Lema 3.1 se caracteriza $L$ definido en (52) en términos de $\rho^{*}$.

Lema $3.1 \mathrm{Sea}$

$$
\rho^{* *}=-\frac{1}{2}\left[\frac{\left(\alpha+\mu_{H}\right)\left[\mu_{H}\left(1-\rho^{*}\right)+\delta\right](1-\theta)}{\alpha \mu_{H}}+\rho_{1}\right] .
$$

$$
\text { 1. } \rho^{* *} \leq \frac{-\rho_{1}}{2} \text {. }
$$

2. Si $\rho^{*}>\rho^{* *}$ entonces $L<1$, mientras que si $\rho^{*} \leq \rho^{* *}$ entonces $L \geq 1$.

Demonstración. 1. Reescribiendo la expresión para $\rho^{* *}$ se obtiene que

$$
\begin{aligned}
\rho^{* *} & =-\frac{1}{2} \frac{\left(\alpha+\mu_{H}\right)\left[\mu_{H}\left(1-\rho^{*}\right)+\delta\right](1-\theta)}{\alpha \mu_{H}}-\frac{\rho_{1}}{2} \\
& <-\frac{\rho_{1}}{2},
\end{aligned}
$$

y si $\theta=1$ entonces $\rho^{* *}=-\rho_{1} / 2$. Por lo tanto $\rho^{* *} \leq-\rho_{1} / 2$.

2. Reescribiendo la expresión para $L$ se tiene que

$$
L=-\frac{2 \alpha \mu_{H}}{\left(\alpha+\mu_{H}\right)\left(\mu_{H}\left(1-\rho^{*}\right)+\delta\right)(1-\theta)} \rho^{*}-\frac{\alpha \mu_{H} \rho_{1}}{\left(\alpha+\mu_{H}\right)\left(\mu_{H}\left(1-\rho^{*}\right)+\delta\right)(1-\theta)} .
$$

Por lo tanto $L$ puede ser visto como una función lineal en la variable $\rho^{*}$ con pendiente negativa, esto es, como una función decreciente de $\rho^{*}$. Por otro lado, la ecuación $L=1$ es equivalente a

$$
\begin{aligned}
\rho^{*} & =-\frac{1}{2}\left[\frac{\left(\alpha+\mu_{H}\right)\left(\mu_{H}\left(1-\rho^{*}\right)+\delta\right)(1-\theta)}{\alpha \mu_{H}}+\rho_{1}\right] \\
& =-\frac{1}{2}\left[\frac{\left(\alpha+\mu_{H}\right)\left[\mu_{H}\left(1-\rho^{*}\right)+\delta\right](1-\theta)}{\alpha \mu_{H}}+\rho_{1}\right] \\
& =\rho^{* *} .
\end{aligned}
$$

Luego, se satisface que si $\rho^{*}>\rho^{* *}$ entonces $L<1$, mientras que si $\rho^{*} \leq \rho^{* *}$ entonces $L \geq 1$.
En las Proposiciones 3.4 y 3.5 se dan condiciones para la existencia de soluciones endémicas del sistema (6) considerando la fuerza de infección para mosquitos $\beta_{1}$. La prueba de estas proposiciones de deduce del análisis de los signos de los coeficientes $a, b$ y $c$ de la ecuación cuadrática (49).

Proposición 3.4 Supóngase que $\rho^{*} \leq \rho^{* *}$.

1. Si $\rho^{*} \leq-\rho_{1}$ entonces

(a) $\mathrm{Si} R_{0}<1$ se tienen las siguientes posibilidades

* Si $R_{0}<R_{0}^{*}$ no existen soluciones de equilibrio endémicas.

* Si $R_{0}=R_{0}^{*}$ existe una única solución de equilibrio endémica.

* Si $R_{0}>R_{0}^{*}$ existen dos soluciones de equilibrio endémicas.

(b) Si $R_{0} \geq 1$ existe una única solución de equilibrio endémica.

2. Si $-\rho_{1}<\rho^{*} \leq-\rho_{1} / 2$ entonces

(a) Si $R_{0} \leq 1$ existe una única solución de equilibrio endémica.

(b) Si $R_{0}>1$ no existen soluciones de equilibrio endémicas.

Proposición 3.5 Supóngase que $\rho^{*}>\rho^{* *}$.

1. Si $\rho^{*} \leq-\rho_{1}$ o $\rho^{*} \geq 0$ entonces

(a) Si $R_{0} \leq 1$ no existen soluciones de equilibrio endémicas.

(b) Si $R_{0}>1$ existe una única solución de equilibrio endémica.

2. Si $-\rho_{1}<\rho^{*}<0$ entonces

(a) Si $R_{0} \leq 1$ existe una única solución de equilibrio endémica.

(b) $\mathrm{Si} R_{0}>1$ existen dos soluciones de equilibrio endémicas.

Nota 3.2 Obsérvese que la Proposición 3.4-1, sugiere la existencia de una bifurcación hacia atrás, mientras que la Proposición 3.4-2, sugiere la existencia de una bifurcación hacia adelante. Por otro lado, en la ecuación (52) si $\rho^{*}=0$ entonces $L<1$, lo cual implica que la condición 1 de la Proposición 3.5 siempre se satisface. Por lo tanto, la Proposición 3.5 muestra que el sistema (6) con $\beta_{1}$ solamente puede exhibir una bifurcación hacia adelante cuando $\rho^{*}=0$.

A continuación se analiza la existencia de soluciones endémicas considerando la fuerza de infección para 
mosquitos $\beta_{2}$ dada en (5). Para este fin, se define el número reproductivo básico $P_{0}$ como

$$
P_{0}=\sqrt{\beta_{h} \beta_{v} \frac{\alpha}{\alpha+\mu_{H}} \frac{1}{\mu_{H}\left(1-\rho^{*}\right)+\delta} \frac{1}{\mu_{V}}}
$$

el cual será calculado en la Sección 4.1. Reemplazando $\beta_{2}$ definido en (5) en la sexta ecuación de (22) se obtiene

$$
I_{H}=\frac{\mu_{V}}{\beta_{v}} \frac{\Lambda_{V}}{\mu_{V}} \frac{I_{V}}{S_{V}}
$$

Reemplazando $I_{H}$ definido en (56) en la tercera y cuarta ecuación de (22) se obtiene que

$$
E_{H}=\frac{\beta_{v}}{\alpha+\mu_{H}} \frac{1}{P_{0}^{2}} \frac{\Lambda_{V}}{\mu_{V}} \frac{I_{V}}{S_{V}}, \quad R_{H}=\frac{\delta}{\omega+\mu_{H}} \frac{\mu_{V}}{\beta_{v}} \frac{\Lambda_{V}}{\mu_{V}} \frac{I_{V}}{S_{V}} .
$$
obtiene

Reemplazando $R_{H}$ y $E_{H}$ definidos en (57) en (31) se

$$
S_{H}=\frac{\Lambda_{H}}{\mu_{H}}-\frac{\beta_{v}}{\mu_{H}}(1-\theta) \frac{1}{P_{0}^{2}} \frac{\Lambda_{V}}{\mu_{V}} \frac{I_{V}}{S_{V}} .
$$

Después de reemplazar $S_{H}, I_{H}, R_{H}$ y $E_{H}$ definidas en (56), (57) y (58), en la población total $N_{H}$ y simplificando

$$
N_{H}=\frac{\Lambda_{H}}{\mu_{H}}+\frac{\rho^{*}}{\rho_{1}} \frac{\Lambda_{V}}{\mu_{V}} \frac{I_{V}}{S_{V}} \frac{\alpha \beta_{h}}{\left(\alpha+\mu_{H}\right)\left(\mu_{H}\left(1-\rho^{*}\right)+\delta\right)} .
$$

Reemplazando $\beta_{H}$ definido en (4) en la segunda ecuación de (22) se obtiene

$$
\frac{\beta_{h} I_{V}}{N_{H}} S_{H}-\left(\alpha+\mu_{H}\right) E_{H}=0
$$

Ahora, reemplazando (57), (58) y (59) en la ecuación (60) se obtiene

$$
\begin{gathered}
-\left(\frac{\beta_{h}}{\mu_{H}}(1-\theta) \frac{1}{P_{0}^{2}}+\frac{1}{S_{V}} \frac{1}{P_{0}^{2}} \frac{\Lambda_{V}}{\mu_{V}} \frac{\rho^{*}}{\rho_{1}} \frac{\alpha \beta_{h}}{\left(\alpha+\mu_{H}\right)\left(\mu_{H}\left(1-\rho^{*}\right)+\delta\right)}\right) \\
\times \frac{I_{V}}{S_{V}}\left(\frac{\mu_{V}}{\Lambda_{V}}-\frac{1}{P_{0}^{2}} \frac{1}{S_{V}}\right) \frac{\Lambda_{H}}{\mu_{H}}=0
\end{gathered}
$$

A partir de la suma de las dos últimas ecuaciones de (22) se obtiene

$$
\frac{I_{V}}{N_{V}}=\frac{\Lambda_{V}}{\mu_{V}} \frac{1}{S_{V}}-1 .
$$

Reemplazando (62) en (61)

$$
\begin{array}{r}
-\left(\frac{\beta_{h}}{\mu_{H}}(1-\theta)+\frac{1}{S_{V}} \frac{\Lambda_{V}}{\mu_{V}} \frac{\rho^{*}}{\rho_{1}} \frac{\alpha \beta_{h}}{\left(\alpha+\mu_{H}\right)\left(\mu_{H}\left(1-\rho^{*}\right)+\delta\right)}\right)\left(\frac{\Lambda_{V}}{\mu_{V}} \frac{1}{S_{V}}-1\right) \\
+\left(\frac{\mu_{V}}{\Lambda_{V}} P_{0}^{2}-\frac{1}{S_{V}}\right) \frac{\Lambda_{H}}{\mu_{H}} \\
=0 .
\end{array}
$$

Multiplicando ambos lados de la igualdad (63) por $S_{V}^{2}$, se obtiene la siguiente ecuación cuadrática para $S_{V}$

$$
\bar{a}\left(S_{V}\right)^{2}-\bar{b} S_{V}-\bar{c}=0
$$

donde

$\bar{a}=\frac{\beta_{h}}{\mu_{H}}(1-\theta)+\frac{\Lambda_{H}}{\mu_{H}} \frac{\mu_{V}}{\Lambda_{V}} P_{0}^{2}$

$\bar{b}=\left[\frac{\beta_{h}}{\mu_{H}}(1-\theta) \frac{\Lambda_{V}}{\mu_{V}}+\frac{\Lambda_{H}}{\mu_{H}}\right]-\frac{\Lambda_{V}}{\mu_{V}} \frac{\rho^{*}}{\rho_{1}} \frac{\alpha \beta_{h}}{\left(\alpha+\mu_{H}\right)\left(\mu_{H}\left(1-\rho^{*}\right)+\delta\right)}$

$\bar{c}=\frac{\rho^{*}}{\rho_{1}} \frac{\alpha \beta_{h}}{\left(\alpha+\mu_{H}\right)\left(\mu_{H}\left(1-\rho^{*}\right)+\delta\right)}\left(\frac{\Lambda_{V}}{\mu_{V}}\right)^{2}$.

Siguiendo un procedimiento similar al realizado para la ecuación cuadrática (49), en la Proposición 3.6 se establecen condiciones para la existencia de soluciones endémicas para el sistema (6) con $\beta_{2}$.

Proposición $3.6 \mathrm{Sea}$

$\eta=\frac{4 \bar{a} \bar{c}}{\bar{b}^{2}}=\frac{\left(\frac{\beta_{h}}{\mu_{H}}(1-\theta)+\frac{\Lambda_{H}}{\mu_{H}} \frac{\mu_{V}}{\Lambda_{V}} P_{0}^{2}\right)\left(\frac{\rho^{*}}{\rho_{1}} \frac{\alpha \beta_{h}}{\left(\alpha+\mu_{H}\right)\left(\mu_{H}\left(1-\rho^{*}\right)+\delta\right)}\left(\frac{\Lambda_{V}}{\mu_{V}}\right)^{2}\right)}{\left[\left[\frac{\beta_{h}}{\mu_{H}}(1-\theta) \frac{\Lambda_{V}}{\mu_{V}}+\frac{\Lambda_{H}}{\mu_{H}}\right]-\frac{\Lambda_{V}}{\mu_{V}} \frac{\rho^{*}}{\rho_{1}} \frac{\alpha \beta_{h}}{\left(\alpha+\mu_{H}\right)\left(\mu_{H}\left(1-\rho^{*}\right)+\delta\right)}\right]^{2}}$.

El sistema (6) siempre tiene un equilibrio trivial libre de infeción $E_{0}$ definido en (23). Además,

1. si $\rho^{*} \geq 0$, entonces existe un único equilibrio endémico.

2. si $\rho^{*}<0$, se presentan la siguientes opciones:

(a) si $0<\eta<1$, entonces existen dos equilibrios endémicos.

(b) si $\eta=1$, entonces existe un único equilibrio endémico.

(c) si $\eta>1$, no existen equilibrios endémicos.

\section{ESTABILIDAD DE LAS SOLUCIONES DE EQUILIBRIO}

En esta sección se analizará la estabilidad de las soluciones de equilibrio del sistema (6).

\subsection{Estabilidad local del equilibrio libre de infección $E_{0}$}

Usando el método de la matriz de la siguiente generación descrito en Driessche y Watmough (2002), se calculan los números reproductivos básicos definidos en las ecuaciones (36) y (55) asociados a $\beta_{i}, i=1,2$ del sistema (6). Además, se probará que la solución de equilibrio libre de infección $E_{0}$ dada en (23) es local y asintóticamente estable si $R_{0}<1$ y $\left(P_{0}<1\right)$, independientemente de la fuerza de infección que se considere. Reorganizando los compartimentos del sistema (6) de la forma

$$
X=\left(E_{H}, I_{H}, I_{V}, S_{H}, S_{V}, R_{H}\right),
$$


en el cual los tres primeros compartimientos corresponden a los individuos infecciosos, se obtiene el sistema

$$
\dot{X}=\mathrm{F}-\mathrm{V},
$$

donde, $\mathrm{F}$ recibe el nombre de matriz de aparición de nuevas infecciones y $\mathrm{V}=\mathrm{V}^{-}-\mathrm{V}^{+}$matriz de transferencia, las cuales vienen dadas por

$$
\begin{aligned}
& \mathrm{F}=\left(\begin{array}{c}
\frac{\beta_{h} I_{V} S_{H}}{N_{H}} \\
0 \\
\beta_{i} S_{V} \\
0 \\
0 \\
0
\end{array}\right), \quad \mathrm{y} \quad \mathrm{V}=\mathrm{V}^{-}-\mathrm{V}^{+}= \\
&\left(\begin{array}{c}
\left(\alpha+\mu_{H}\right) E_{H} \\
\left(\rho+\delta+\mu_{H}\right) I_{H} \\
\mu_{V} I_{V} \\
\mu_{H} S_{H}+\frac{\beta_{h} I_{V} S_{H}}{N_{H}} \\
\mu_{V} S_{V}+\frac{\beta_{v} I_{H} S_{V}}{N_{H}} \\
\left(\omega+\mu_{H}\right) R_{H}
\end{array}\right)-\left(\begin{array}{c}
\left(\alpha+\mu_{H}\right) E_{H} \\
0 \\
\lambda I_{H}+\alpha E_{H} \\
0 \\
\omega R_{H}+\Lambda_{H} \\
\Lambda_{V} \\
\delta I_{H}
\end{array}\right)=\left(\begin{array}{c}
\left(\rho+\delta+\mu_{H}\right) I_{H}-\left(\lambda I_{H}+\alpha E_{H}\right) \\
\mu_{V} I_{V} \\
\mu_{H} S_{H}+\frac{\beta_{h} I_{V} S_{H}}{N_{H}}-\left(\omega R_{H}+\Lambda_{H}\right) \\
\mu_{V} S_{V}+\frac{\beta_{v} I_{H} S_{V}}{N_{H}}-\Lambda_{V} \\
\left(\omega+\mu_{H}\right) R_{H}-\delta I_{H}
\end{array}\right) .
\end{aligned}
$$

Obsérvese además que

$$
X_{i}=\mathrm{F}_{i}-\mathrm{V}_{i}=f_{i}, \quad i=1, \ldots, 6 .
$$

Denótese por $X_{s}$ al conjunto de todos los equilibrios libres de infección del sistema (6), entonces

$$
\begin{gathered}
X_{s}=\left\{\left(X_{1}, \ldots, X_{6}\right) \in R_{+}^{6}: X_{i}=0, \quad \text { para } i=1,2,3\right\} \\
=\left\{E_{0}\right\} .
\end{gathered}
$$

A partir del Lema 1. de Driessche y Watmough (2002) se establece que la matriz jacobiana $D f\left(E_{0}\right)$ del sistema (6) se puede reescribir de la siguiente manera

$$
D f\left(E_{0}\right)=D \mathrm{~F}\left(E_{0}\right)-D \mathrm{~V}\left(E_{0}\right),
$$

donde

$$
D \mathrm{~F}\left(E_{0}\right)=\left(\begin{array}{cc}
F & 0 \\
0 & 0
\end{array}\right) \text { y } D \mathrm{~V}\left(E_{0}\right)=\left(\begin{array}{cc}
V & 0 \\
J_{3} & J_{4}
\end{array}\right),
$$

siendo $F$ y $V$ matrices $3 \times 3$ definidas por

$$
F=\left(\frac{\partial \mathrm{F}_{i}}{\partial X_{j}}\left(E_{0}\right)\right) \text { y } V=\left(\frac{\partial \mathrm{V}_{i}}{\partial X_{j}}\left(E_{0}\right)\right) \text { para } 1 \leq i, j \leq 3 .
$$

Más aún, a partir del Lema 1. de Driessche y Watmough (2002) se concluye que $F$ es una matriz no negativa, $V$ es una $M$-matriz no singular y todos los valores propios de $J_{4}$ tienen parte real positiva. En consecuencia, para el sistema (6) se obtienen $F_{i}, V_{i}$ y $J_{4}^{i}$ para cada $\beta_{i}$ las cuales vienen dadas por

$$
F_{i}=\left(\begin{array}{ccc}
0 & 0 & \beta_{h} \\
0 & 0 & 0 \\
0 & \beta_{i}^{*} & 0
\end{array}\right) \quad i=1,2
$$

Donde

$$
\beta_{1}^{*}=\beta_{v} \frac{\Lambda_{V} \mu_{H}}{\Lambda_{H} \mu_{V}}, \beta_{2}^{*}=\beta_{v} .
$$

Además

$$
V_{1}=V_{2}=V=\left(\begin{array}{ccc}
\alpha+\mu_{H} & 0 & 0 \\
-\alpha & \mu_{H}\left(1-\rho^{*}\right)+\delta & 0 \\
0 & 0 & \mu_{V}
\end{array}\right),
$$

y

$$
J_{4}^{1}=J_{4}^{2}=J_{4}=\left(\begin{array}{ccc}
\mu_{H} & 0 & \\
0 & \mu_{V} & 0 \\
0 & 0 & \omega+\mu_{H}
\end{array}\right) .
$$

Debido a la no singularidad de la matriz $V$, nótese que su matriz inversa viene dada por

$$
V^{-1}=\left(\begin{array}{ccc}
\frac{1}{\alpha+\mu_{H}} & 0 & 0 \\
\frac{\alpha}{\left(\alpha+\mu_{H}\right)\left(\mu_{H}\left(1-\rho^{*}\right)+\delta\right)} & \frac{1}{\mu_{H}\left(1-\rho^{*}\right)+\delta} & 0 \\
0 & 0 & \frac{1}{\mu_{V}}
\end{array}\right) \text {. }
$$

Por lo tanto, según Driessche y Watmough (2002) se verifica que la matriz de la siguiente generación en cada uno de los casos viene dada por

$$
P_{i}=F_{i} V^{-1}=\left(\begin{array}{ccc}
0 & 0 & \frac{\beta_{h}}{\mu_{V}} \\
0 & 0 & 0 \\
P_{13}^{i} & P_{23}^{i} & 0
\end{array}\right),
$$

donde

$$
\begin{gathered}
P_{13}^{1}=\frac{\alpha \beta_{v}}{\left(\alpha+\mu_{H}\right)\left(\mu_{H}\left(1-\rho^{*}\right)+\delta\right)} \frac{\Lambda_{V} \mu_{H}}{\Lambda_{H} \mu_{V}}, P_{13}^{2}=\frac{\alpha \beta_{v}}{\left(\alpha+\mu_{H}\right)\left(\mu_{H}\left(1-\rho^{*}\right)+\delta\right)} \\
P_{23}^{1}=\frac{\beta_{v}}{\mu_{H}\left(1-\rho^{*}\right)+\delta} \frac{\Lambda_{V} \mu_{H}}{\Lambda_{H} \mu_{V}}, P_{23}^{2}=\frac{\beta_{v}}{\mu_{H}\left(1-\rho^{*}\right)+\delta} .
\end{gathered}
$$

Un valor propio de la matriz $P_{i}, i=1,2$ es $\xi_{1}^{i}=0$, mientras que para $P_{1}$ los demás valores propios vienen dados por la solución de la ecuación

$$
\xi^{2}-\beta_{h} \beta_{v} \frac{\Lambda_{V} \mu_{H}}{\Lambda_{H} \mu_{V}} \frac{\alpha}{\mu_{V}\left(\alpha+\mu_{H}\right)\left(\mu_{H}\left(1-\rho^{*}\right)+\delta\right)}=0 .
$$

Por lo tanto, el radio espectral de la matriz $P_{1}$ denotado como $R_{0}$ es el número reproductivo básico definido en (36). 
Para la matriz $P_{2}$ los demás valores propios vienen dados por la solución de la ecuación

$$
\xi^{2}-\frac{\alpha \beta_{h}}{\left(\mu_{H}\left(1-\rho^{*}\right)+\delta\right)\left(\mu_{H}+\alpha\right)} \frac{\beta_{v}}{\mu_{V}}=0,
$$

de donde se obtiene el número reproductivo básico $P_{0}$ definido en (55).

A continuación se prueba la estabilidad asintótica local del equilibrio libre de infección $E_{0}$.

Proposición 4.1 Si $R_{0}<1$ o $P_{0}<1$, el equilibrio libre de infección $E_{0}$ definido en (23) del sistema (6) es local asintóticamente estable en $\Omega$ definido en (7). Si $R_{0}>10$ $P_{0}>1$ entonces $E_{0}$ es inestable.

Demostración. Basta verificar que el sistema (6) satisface las hipótesis $\left(A_{1}\right)-\left(A_{5}\right)$ de [11], donde el contador $j$ representa un compartimiento del sistema reorganizado (67).

1. $\left(A_{1}\right)$ : Si $X \geq 0$ entonces $\mathrm{F}_{j}, \mathrm{~V}_{j}^{+} \mathrm{y} \mathrm{V}_{j}^{-} \geq 0 \quad \forall j=1, \ldots, 6$. En efecto, pues cada función representa una transferencia directa de individuos (humanos y mosquitos).

2. $\left(A_{2}\right)$ : Si $X_{j}=0$ entonces $\bigvee_{j}^{-}=0 \quad \forall j=1,2,3$. En efecto, si $E_{H}=I_{H}=I_{V}=0$, entonces $\mathrm{V}_{1}^{-}, \mathrm{V}_{2}^{-}$y $\mathrm{V}_{3}^{-}$son todas iguales a cero.

3. $\left(A_{3}\right): \mathrm{F}_{j}=0 \quad \forall j>3$. De (68) se observa que $\mathrm{F}_{4}=\mathrm{F}_{5}=\mathrm{F}_{6}=0$.

4. $\left(A_{4}\right)$ : Si $X \in X_{s}$ entonces $\mathrm{F}_{j}(X)=0$ y $\mathrm{V}_{j}^{+}=0 \quad \forall j=1,2,3$. Puesto que $X_{s}=\left\{E_{0}\right\}$, entonces de (71) se observa que $\mathrm{F}_{1}\left(E_{0}\right)=\mathrm{F}_{2}\left(E_{0}\right)=\mathrm{F}_{3}\left(E_{0}\right)=\mathrm{V}_{1}^{+}\left(E_{0}\right)=\mathrm{V}_{2}^{+}\left(E_{0}\right)=\mathrm{V}_{3}^{+}\left(E_{0}\right)=0$.

5. $\left(A_{5}\right)$ : En una vecindad del cero todos los valores propios de $D f\left(E_{0}\right)$ tienen parte real negativa. Para verificar esta propiedad basta notar que de (70) el conjunto de valores propios de $D f\left(E_{0}\right)$ está particionado en el conjunto de valores propios de la matriz $F_{i}-V$ y la matriz $-J_{4}$. Puesto que la parte real de los valores propios de $J_{4}$ son positivos, entonces la parte real de los valores propios de $-J_{4}$ tienen parte real negativa. Luego el signo de la parte real de los valores propios de $D f\left(E_{0}\right)$ está caracterizado por el signo de la parte real de los valores propios de $F_{i}-V$. Sea

$$
J_{1}^{i}=F_{i}-V .
$$

El Teorema 2. de Driessche y Watmough (2002) garantiza que $s\left(J_{1}\right)<0$ si y sólo si $R_{0}, P_{0}<1$, donde $s\left(J_{1}^{i}\right)$ denota la abscisa espectral de la matriz $J_{1}^{i}$ (el máximo de la parte real de todos los valores propios de $J_{1}^{i}$ ). Puesto que la parte real de los demás valores propios de $J_{1}^{i}$ es menor que $s\left(J_{1}^{i}\right)$, entonces se concluye que la parte real de todos los valores propios de la matriz $J_{1}^{i}$ tienen parte real negativa si y sólo si $R_{0}, P_{0}<1$.

\subsection{Estabilidad global del equilibrio libre de infección}

A continuación, usando el principio de invarianza de Lasalle y el Teorema de Lyapunov (Perko, 1991), se prueba la estabilidad asintótica global de la solución de equilibrio libre de infección $E_{0}$. La Proposición 4.2 se plantea para la fuerza de infección de mosquitos $\beta_{1}$, el caso $\beta_{2}$ es análogo y se enuncia en la Proposición 4.3.

Proposición 4.2 Si $\rho^{*} \leq 0$ y $R_{0}^{2}<\ell$, entonces el equilibrio trivial libre de infección $E_{0}$ es global asintóticamente estable en $\Omega$ donde,

$$
\ell=1+\frac{\beta_{h} \beta_{v} \alpha \Lambda_{V} \mu_{H} \rho^{*}}{\Lambda_{H} \mu_{V}^{2}\left(\alpha+\mu_{H}\right)\left[\mu_{H}\left(1-\rho^{*}\right)+\delta\right]} .
$$

Demostración. Por la Proposición 4.1, se sigue que $E_{0}$ es local asintóticamente estable en $\Omega$ cuando $R_{0}^{2}<1$. Defínase

$$
R_{*}=\frac{\beta_{h} \beta_{v} \alpha \Lambda_{V} \mu_{H}}{\Lambda_{H} \mu_{V}^{2}\left(\alpha+\mu_{H}\right)\left[\mu_{H}\left(1-\rho^{*}\right)+\delta\right]} .
$$

Mediante cálculos sencillos se verifica que

$$
R_{*}=R_{0}^{2}+1-\ell .
$$

Ahora bien, supóngase que $R_{0}^{2}<\ell$, lo cual por (80) es equivalente a suponer que $R_{*}<1$, y sea $\left(S_{H}(t), E_{H}(t), I_{H}(t), R_{H}(t), S_{V}(t), I_{V}(t)\right)$ una solución positiva del sistema (6) para la cual $N_{V}(t)=S_{V}(t)+I_{V}(t)$, entonces por hipótesis del Lema 2.1 se sigue que

$$
N_{V}(t) \leq \frac{\Lambda_{V}}{\mu_{V}} .
$$

Puesto que $I_{H}(t)<N_{H}(t)$ y $\rho^{*} \leq 0$, entonces

$$
\begin{aligned}
\dot{N}_{H}(t) & =\Lambda_{H}-\mu_{H} N_{H}+\mu_{H} \rho^{*} I_{H} \\
& >\Lambda_{H}-\mu_{H} N_{H}+\mu_{H} \rho^{*} N_{H} \\
& =\Lambda_{H}+\mu_{H}\left(1-\rho^{*}\right) N_{H} .
\end{aligned}
$$

Luego

$$
N_{H}(t)>\frac{\Lambda_{H}}{\mu_{H}\left(1-\rho^{*}\right)} .
$$

Se probará la existencia de una función de Lyapunov para el sistema trasladado

$$
\dot{x}=F\left(x+E_{0}\right)-F\left(E_{0}\right)=f(x),
$$

donde el sistema

$$
\dot{y}=F(y)
$$

tiene a $y=0$ como solución de equilibrio y $F$ representa el campo vectorial definido por el lado izquierdo del sistema (6). Considérese la siguiente función: 


$$
V^{*}\left(S_{H}, E_{H}, I_{H}, R_{H}, S_{V}, I_{V}\right)=I_{V}+\frac{\mu_{V}}{\beta_{h}} E_{H}+\frac{\mu_{V}\left(\alpha+\mu_{H}\right)}{\alpha \beta_{h}} I_{H},
$$

y defínase

$$
V\left(\tilde{S}_{H}, \tilde{E}_{H}, \tilde{I}_{H}, \tilde{R}_{H}, \tilde{S}_{V}, \tilde{I}_{V}\right) \mathrm{B} V^{*}\left(S_{H}-\frac{\Lambda_{H}}{\mu_{H}}, E_{H}, I_{H}, R_{H}, S_{V}-\frac{\Lambda_{V}}{\mu_{V}}, I_{V}\right) .
$$

A continuación se prueba que la función $V$ definida en (81) es una función de Lyapunov. En efecto, $V$ es definida positiva pues $V\left(E_{0}\right)=V^{*}(\mathbf{0})=0 \quad$ y $\quad V>0$ $\forall\left(\tilde{S}_{H}, \tilde{E}_{H}, \tilde{I}_{H}, \tilde{R}_{H}, \tilde{S}_{V}, \tilde{I}_{V}\right) \neq E_{0} \in \Omega$, y además la derivada orbital de $V$ a lo largo de las trayectorias de (6) viene dada por

$$
\begin{aligned}
\dot{V} & =\frac{\partial V}{\partial \tilde{S}_{H}} f_{1}+\ldots+\frac{\partial V}{\partial \tilde{I}_{V}} f_{6} \\
& =\frac{\partial V^{*}}{\partial\left(S_{H}-\Lambda_{H} / \mu_{H}\right)} f_{1}+\ldots+\frac{\partial V^{*}}{\partial\left(S_{V}-\Lambda_{V} / \mu_{V}\right)} f_{5}+\frac{\partial V^{*}}{\partial I_{V}} f_{6} \\
& =\dot{I}_{V}+\frac{\mu_{V}}{\beta_{h}} \dot{E}_{H}+\frac{\mu_{V}\left(\alpha+\mu_{H}\right)}{\alpha \beta_{h}} \dot{I}_{H} \\
& =\frac{\beta_{v}}{N_{H}} I_{H} S_{V}-\mu_{V} I_{V}+\frac{\mu_{V}}{\beta_{h}}\left[\frac{\beta_{h}}{N_{H}} I_{V} S_{H}-\left(\alpha+\mu_{H}\right) E_{H}\right]- \\
& \frac{\mu_{V}\left(\alpha+\mu_{H}\right)}{\alpha \beta_{h}}\left[\left(\mu_{H}\left(1-\rho^{*}\right)+\delta\right) I_{H}+\alpha E_{H}\right] \\
& =\left[\frac{\beta_{v}}{N_{H}} S_{V}-\frac{\mu_{V}\left(\alpha+\mu_{H}\right)\left[\mu_{H}\left(1-\rho^{*}\right)+\delta\right]}{\alpha \beta_{h}}\right] I_{H}-\left[\frac{N_{H}-S_{H}}{N_{H}}\right] I_{V} \\
& \leq\left[\frac{\beta_{v}}{N_{H}} S_{V}-\frac{\mu_{V}\left(\alpha+\mu_{H}\right)\left[\mu_{H}\left(1-\rho^{*}\right)+\delta\right]}{\alpha \beta_{h}}\right] I_{H} \\
& {\left[\begin{array}{l}
\beta_{v}\left(\frac{\Lambda_{V}}{\mu_{V}}\right) \\
\Lambda_{H}
\end{array}-\frac{\mu_{V}\left(\alpha+\mu_{H}\right)\left[\mu_{H}\left(1-\rho^{*}\right)+\delta\right]}{\alpha \beta_{h}}\right] I_{H} } \\
& =\left[\frac{\Lambda_{H} \mu_{V}^{2}\left(\alpha+\mu_{H}\right)\left[\mu_{H}\left(1-\rho^{*}\right)+\delta\right]\left(1-\rho^{*}\right)}{\beta_{h} \alpha \Lambda_{H} \mu_{V}} \times\left(R_{*}-1\right)\right] I_{H} .
\end{aligned}
$$

Por el Lema 2.1 el primer factor es positivo y por hipótesis se tiene que $R_{*}<1$. Entonces $\dot{V} \leq 0, \forall I_{H} \geq 0$ y $\forall I_{V} \geq 0$. Sea

$$
\Delta=\left\{\left(S_{H}, E_{H}, I_{H}, R_{H}, S_{V}, I_{V}\right): \dot{V}=0\right\},
$$

entonces

$\Delta \subset\left\{\left(S_{H}, E_{H}, I_{H}, R_{H}, S_{V}, I_{V}\right): I_{H}=0\right\} \cup\left\{\left(S_{H}, E_{H}, I_{H}, R_{H}, S_{V}, I_{V}\right): I_{V}=0\right\}$ Sea $\Delta^{\prime} \subset \Delta$ el mayor conjunto invariante con respecto (6) y sea

$$
\left(S_{H}, E_{H}, I_{H}, R_{H}, S_{V}, I_{V}\right)
$$

una solución de (6) en $\Delta^{\prime}$, entonces $\left(S_{H}, E_{H}, I_{H}, R_{H}, S_{V}, I_{V}\right)$ está definida $\mathrm{y}$ es acotada $\forall t \in \mathrm{R}$. Puesto que $\Delta^{\prime} \subset\left\{\left(S_{H}, E_{H}, I_{H}, R_{H}, S_{V}, I_{V}\right): I_{H}=0\right\} \quad$ entonces $I_{H}(t) \equiv 0$, y reemplazando en el sistema (6) se obtiene que $E_{H}(t) \equiv R_{H}(t) \equiv I_{V}(t) \equiv 0$, mientras que de la primera y quinta ecuación de (6) se obtiene que $S_{H}(t) \equiv \Lambda_{H} / \mu_{H}$ y $S_{V}(t) \equiv \Lambda_{V} / \mu_{V}$. Por tanto $\Delta^{\prime}=\left\{E_{0}\right\}$. Por principio de invarianza de LaSalle, $E_{0}$ es un atractor global, es decir $E_{0}$ es global asintóticamente estable cuando $R_{0}^{2}<\ell$.
Corolario $4.1 \mathrm{Si} \rho^{*}=0$ y $R_{0}<1$, entonces el equilibrio libre de infección $E_{0}$ del sistema (6) es globalmente asintóticamente estable.

Demostración. Basta observar que de la definición de $\ell$ que cuando $\rho^{*}$ entonces $\ell=1$.

Para el caso de la fuerza de infección de los mosquitos $\beta_{2}$ se tiene la siguiente proposición.

Proposición 4.3 Si $P_{0}<1$, entonces el equilibrio trivial libre de infección $E_{0}$ es global asintóticamente estable en $\Omega$.

Demostración. Considerando la función de Lyapunov definida (81) y usando el hecho de que

$$
\frac{S_{V}}{N_{V}} \leq 1
$$

se verifica que la derivada orbital de $V$ sobre el sistema (6) es no negativa.

4.3 Estabilidad de las soluciones de equilibrio endémicas para el caso constante

A continuación, se analizará la estabilidad asintótica local de las soluciones de equilibrio endémicas del sistema (6) para $\beta_{i}, i=1,2$ constantes. Se iniciará analizando la estabilidad local de los equilibrios endémicos a través de la linealización del sistema (6) alrededor del equilibrio endémico $E$, la cual está dada por $\dot{X}=J(E) X$, donde $X=\left(S_{H}, E_{H}, I_{H}, R_{H}, S_{V}, I_{V}\right)$ y la matriz jacobiana $J$ evaluada en $E$ está dada por

$$
J(E)=\left(\begin{array}{cccccc}
j_{11} & j_{12} & j_{12} & j_{14} & 0 & j_{16} \\
j_{21} & -j_{22} & -j_{12} & -j_{12} & 0 & -j_{16} \\
0 & \alpha & -j_{33} & 0 & 0 & 0 \\
0 & 0 & \delta & -j_{44} & 0 & 0 \\
-j_{51} & -j_{52} & -j_{53} & -j_{54} & -j_{55} & -j_{56} \\
j_{51} & j_{52} & j_{53} & j_{54} & j_{65} & j_{66}
\end{array}\right),
$$

Donde

$$
\begin{array}{ll}
j_{11}(E)=\frac{\beta_{H} S_{H}}{N_{H}}-\left(\beta_{H}+\mu_{H}\right) \quad j_{12}(E)=\frac{\beta_{H} S_{H}}{N_{H}} & j_{14}(E)=\omega+\frac{\beta_{H} S_{H}}{N_{H}} \\
j_{16}(E)=\frac{\beta_{H} S_{H}}{I_{V}} \quad j_{21}(E)=\beta_{H}\left(1-\frac{S_{H}}{N_{H}}\right) & j_{22}(E)=\frac{\beta_{H} S_{H}}{N_{H}}+\alpha+\mu_{H} \\
j_{33}(E)=\frac{\alpha E_{H}}{I_{H}} & j_{44}(E)=\omega+\mu_{H} \quad j_{51}(E)=\frac{d \beta_{i}}{d S_{H}} S_{V} \\
j_{52}(E)=\frac{d \beta_{i}}{d E_{H}} S_{V} \quad j_{53}(E)=\frac{d \beta_{i}}{d I_{H}} S_{V} \quad j_{54}(E)=\frac{d \beta_{i}}{d R_{H}} S_{V} \\
j_{55}(E)=\frac{d \beta_{i}}{d S_{V}} S_{V}+\left(\beta_{i}+\mu_{V}\right) \quad j_{56}(E)=-\frac{d \beta_{i}}{d I_{V}} S_{V} \quad j_{65}(E)=\frac{d \beta_{i}}{d S_{V}} S_{V}+\beta_{i} \\
j_{66}(E)=\frac{d \beta_{i}}{d I_{V}} S_{V}-\mu_{V}, \quad \text { para } \quad i=1,2 .
\end{array}
$$

Obsérvese que para $\beta_{1}$ constante, se satisface que

$$
\begin{aligned}
0 & =j_{51}(E)=j_{52}(E)=j_{53}(E)=j_{54}(E)=j_{56}(E) \\
j_{55}(E) & =-\left(\beta_{1}+\mu_{V}\right)
\end{aligned}
$$




$$
\begin{aligned}
& j_{65}(E)=\beta_{1} \\
& j_{66}(E)=-\mu_{V} .
\end{aligned}
$$

En este caso se verifica que los valores propios de $J(E)$ definida en (82) están dados por

$$
\kappa_{1}=-\left(\beta_{1}+\mu_{V}\right), \quad \kappa_{2}=-\mu_{V},
$$

y las raíces del polinomio de grado cuatro

$$
\kappa^{4}+a_{1} \kappa^{3}+a_{2} \kappa^{2}+a_{3} \kappa+a_{4}=0,
$$

donde

$$
\begin{aligned}
a_{1}= & -j_{11}+j_{22}+j_{33}+j_{44} \\
a_{2}= & -j_{11}\left(j_{22}+j_{33}+j_{44}\right)+j_{22}\left(j_{33}+j_{44}\right)+j_{33} j_{44}+\alpha j_{12}-j_{21} j_{12} \\
a_{3}= & -j_{11}\left(j_{22} j_{33}+j_{22} j_{44}+j_{33} j_{44}\right)+j_{22} j_{33} j_{44}+\alpha j_{12}\left(-j_{11}+j_{44}+\delta\right) \\
& -j_{21} j_{12}\left(j_{33}+j_{44}\right)-\alpha j_{21} j_{12} \\
a_{4}= & -\left(j_{11} j_{22}+j_{12} j_{21}\right) j_{33} j_{44}-\alpha \delta\left(j_{11} j_{12}+j_{14} j_{21}\right)-\alpha j_{12}\left(j_{11} j_{44}+j_{21} j_{44}\right) .
\end{aligned}
$$

El criterio de Routh-Hurwitz [13] establece que si $a_{4}>0$, $D_{1}=a_{1}>0, \quad D_{2}=a_{1} a_{2}-a_{3}>0 \quad$ y $\quad D_{3}=a_{1}\left(a_{2} a_{3}-a_{1} a_{4}\right)-a_{3}^{2}>0$, entonces las raíces de la ecuación (84) tienen parte real negativa. A partir de los componentes $j_{r s}$ definidos en (83) se obtienen la siguientes desigualdades

$$
-j_{11}>0,-j_{11}>j_{21}, j_{22}>j_{12}+\alpha, j_{44}>j_{14} \mathrm{y} j_{33}>\delta .
$$

Después de algunos cálculos y simplifaciones, y además utilizando las desigualdades definidas en (85) se verifica que

$$
\begin{aligned}
a_{4}> & j_{14}\left[-j_{11} j_{21}-j_{21}\left(j_{12}+\alpha\right)\right]>0 \\
D_{1}= & a_{1}>0 \\
D_{2}= & a_{1} a_{2}-a_{3} \\
= & \left(j_{11}\right)^{2}\left(j_{21}+j_{22}+j_{33}+j_{44}\right)+\alpha_{H} j_{12}\left(j_{21}+j_{33}-\delta\right) \\
& +\left(j_{33}+j_{44}\right)\left[j_{33} j_{44}+\left(-j_{11}+j_{22}\right)\left(j_{33}+j_{44}\right)\right]>0 .
\end{aligned}
$$

Siguiendo un procedimiento similar se verifica que $a_{2} a_{3}-a_{1} a_{4}>0$. Además, multiplicando la desigualdad anterior por $a_{1}$ y repitiendo el procedimiento se verifica que $D_{3}>0$. En consecuencia, todos los valores propios de $J(E)$ tienen parte real negativa lo cual implica la estabilidad local del equilibrio endémico $E$. Los resultados anteriores se resumen en la siguiente proposición.

Proposición 4.4 Si $\beta_{1}$ es constante, entonces el equilibrio endémico $E$ es local asintóticamente estable en $\Omega$ cuando $R_{1}>1$.

Siguiendo un procedimiento similar se obtienen resultados análogos para el equilibrio endémico $\tilde{E}$ cuando $\beta_{2}$ constante y para el equilibrio endémico $E^{*}$ cuando $\beta_{H}$ es constante.

Nota 4.1 A partir de los resultados teóricos anteriores se

verifica la existencia de una bifurcación hacia adelante cuando alguna de las fuerzas de infección es constante; es decir, el equilibrio libre de infección estable se bifurca en un equilibrio endémico estable cuando el número reproductivo básico es uno.

\section{SIMULACIONES NUMÉRICAS}

Los valores de los parámetros descritos a continuación fueron tomados de los informes presentados por el Sistema Nacional de Vigiliancia en Salud Pública (SIVIGILA) en el Pacífico Nariñense, durante el periodo comprendido entre finales del año 2000 y el año 2001, periodo en el cual se registró un crecimiento acelerado de casos de malaria en los municipios ubicados en la costa pacífica nariñense. Dentro de la misma zona se pudo observar un dinamismo de la enfermedad, lo cual provocó que ciertos municipios presenten un mayor riesgo que otros. Por tal razón, al estratificar el riesgo por municipios y sectores en el año 2001, se permitió hacer un seguimiento más cercano del comportamiento de la enfermedad con el fin de precisar en las medidas de control a tomar. Los rangos para los valores de los parámetros fueron tomados de Chitnis (2005).

A continuación se caracterizan dos conjuntos de valores para los parámetros del modelo (6): el conjunto de parámetros para zonas de transmisión alta y zonas de transmisión baja en San Andrés de Tumaco-Nariño (SAT). Cuando la fuerza de infección para mosquitos considerada es $\beta_{1}$, se tiene que en zonas de transmisión alta $R_{0}=8.04$ y las soluciones de equilibrio tienden al equilibrio endémico $E=$ (8.621, 2.703, 69.717, 18.236, 8.587, 36.997), mientras que en zonas de transmisión baja $R_{0}=0.56$ y las soluciones tienden al equilibrio endémico $E=(5.230,1.242,10.720,3.171,22.040$, 41.660). Cuando la fuerza de infección es $\beta_{2}$, en zonas de transmisión alta $P_{0}=5.99$ y las soluciones tienden al equilibrio endémico $E=(7.814,2.729,70.391,18.415,4.346$, 41.238) y en zonas de transmisión baja $P_{0}=0.42$ y las soluciones tienden al equilibrio trivial $E_{0}$ definido en (23).

En la Tabla 2 se muestran los valores asignados para los parámetros descritos en la Tabla 1, los cuales están medidos en días como unidad de tiempo.

Tabla 2. Valores de los parámetros del sistema (6) con unidad de tiempo días. La descripción de los parámetros se encuentra en el Cuadro 1.

\begin{tabular}{ccc}
\hline Parámetros & Zonas transmisión alta & Zonas transmisión baja \\
\hline$\alpha$ & 0.10 & 0.10 \\
$\mu_{V}$ & 0.0039 & 0.0025 \\
$\mu_{H}$ & 0.00102 & 0.00125 \\
$\rho$ & 0.0090 & 0.009 \\
$\delta$ & 0.0029 & 0.0029 \\
$\beta_{H V}$ & 0.70 & 0.40 \\
$\beta_{V H}$ & 0.20 & 0.32 \\
$\varepsilon$ & 0.45 & 0.29 \\
$\phi$ & 0.27 & 0.10 \\
$\lambda$ & 0.0091 & 0.0002 \\
$\Lambda_{V}$ & 180 & 160 \\
$\Lambda_{H}$ & 100 & 90 \\
$\omega$ & 0.01 & 0.01 \\
\hline
\end{tabular}



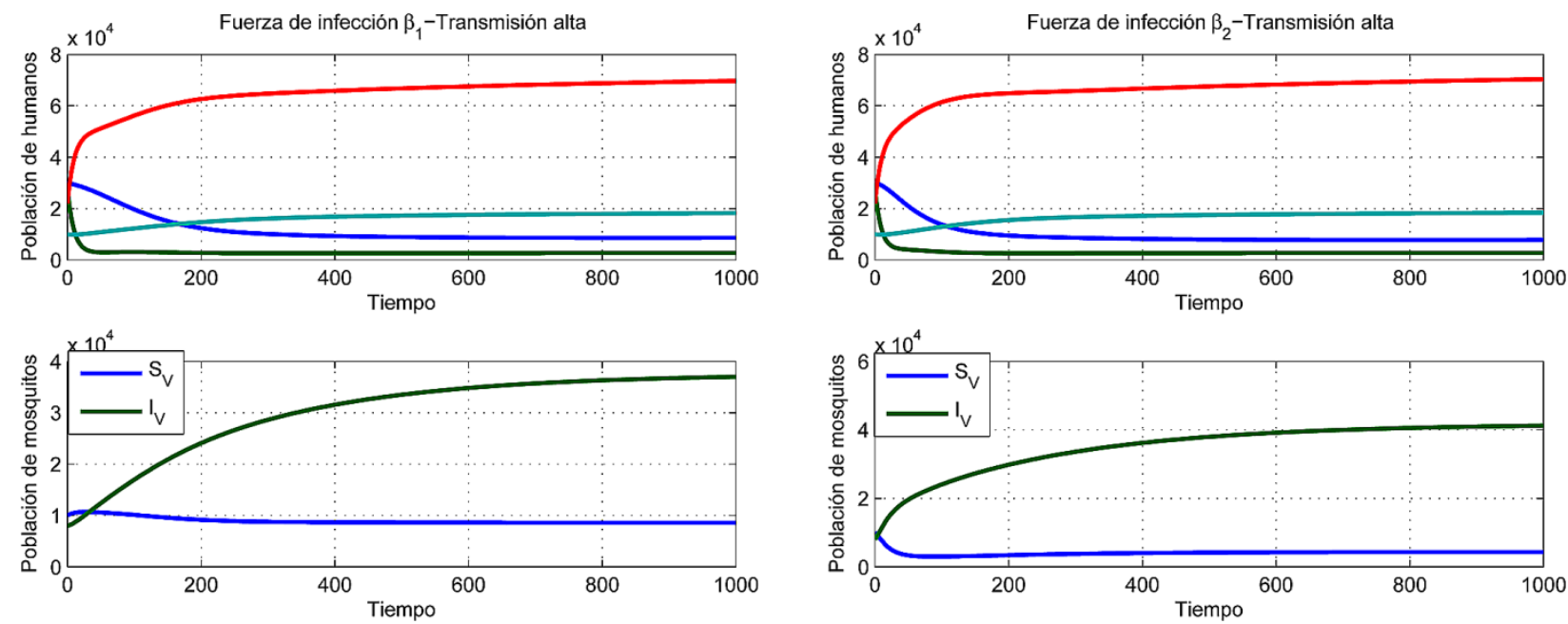

Figura 1. Gráficas para las simulaciones numéricas del sistema (6) en zonas de transmisión alta de SAT. A la izquierda considerando la fuerza de infección para mosquitos $\beta_{1}$ y a la derecha considerando $\beta_{2}$. En este escenario con $\beta_{1}$ se tiene que $R_{0}=8.04$ y las soluciones tienden al equibrio $E=(8.621,2.703,69.717,18.236,8.587,36.997)$. Con $\beta_{2}$ se tiene que $P_{0}=5.99$ y las soluciones tienden al equilibrio $E=(7.814,2.729$, $70.391,18.415,4.346,41.238)$. Las condiciones iniciales fueron $(30.000,28.000,22.000,10.000,10.000,8.00)$. Las simulaciones fueron realizadas usando ode 45 de MATLAB.

De la Figura 1 obtenida con datos de zonas de transmisión alta en SAT, se puede observar que cuando en el sistema (6) se considera la fuerza de infección de los mosquitos $\beta_{2}$ se obtiene un leve aumento de los casos de humanos infectados y una disminución considerable de los casos de mosquitos infectados, con respecto a los resultados obtenidos con la fuerza de infección $\beta_{1}$, lo cual sugiere que con la inclusión de estrategias de control en el modelo, la erradicación de la infección se obtendría más fácilmente considerando la fuerza de infección $\beta_{2}$. La afirmación anterior es corroborada si se observa la Figura 2, pues para zonas de transmisión baja en SAT, con la fuerza de infección de los mosquitos $\beta_{1}$ se observa que las soluciones tienden a un equilibrio endémico, mientras que cuando se considera $\beta_{2}$ se obtiene la solución de equilibrio libre de infección. Lo anterior resulta evidente, teniendo en cuenta que para el modelo que considera $\beta_{2}$ no existe una bifurcación hacia atrás.

\section{DISCUSIÓN |}

En este trabajo se modeló la dinámica de transmisión de la malaria considerando la interacción entre la población humana $N_{H}$ en los estadíos de susceptibles, expuestos, infectados y recuperados de la enfermedad, y la población de mosquitos $N_{V}$ en los estadíos de susceptibles e infectados con Plasmodium, el párasito que ocasiona la enfermedad de malaria. La transmisión de la enfermedad fue modelada de forma horizontal, es decir por picadura del mosquito, y de forma vertical, esto es, de madre infectada a hijo. Se propusieron dos modelos distintos: uno cuando la fuerza de infección del mosquito depende de la población total humana, la cual es clásica en la literatura, y el otro cuando la fuerza de infección de los mosquitos depende de la población total de los mosquitos, la cual no se ha encontrado aún en referencias.

Los resultados del análisis cualitativo del modelo evidenciaron la existencia de una única solución de equilibrio libre de infección $E_{0}$, cuya existencia es independiente de la fuerza de infección considerada. Se probó, usando el número reproductivo básico para cada caso, que la solución de equilibrio libre de infección es local y asintóticamente estable, siempre y cuando el número reproductivo básico se mantenga por debajo de uno; con condiciones adicionales se logró probar la estabilidad asintótica global del equilibrio libre de infección. Lo anterior sugiere que cuando el número de infecciones secundarias producidas por la introducción de un individuo infectado a una población susceptible es menor que uno, la enfermedad de malaria puede controlarse empleando esfuerzos mínimos.

Otro resultado interesante, hace referencia a la dependencia de los números reproductivos básicos a los parámetros. En la literatura se encuentra que el número reproductivo básico no depende explícitamente del período de latencia de los humanos $\alpha$, mientras que en el presente trabajo se encontró que el número reproductivo básico para cada una de las fuerzas de infección consideradas, depende explícitamente del parámetro $\alpha$, cuya inclusión según Mandal (2011) se ha demostrado que no solamente reduce a largo plazo la prevalencia de individuos infectados, sino que también la tasa de progresión a dicho estado. 

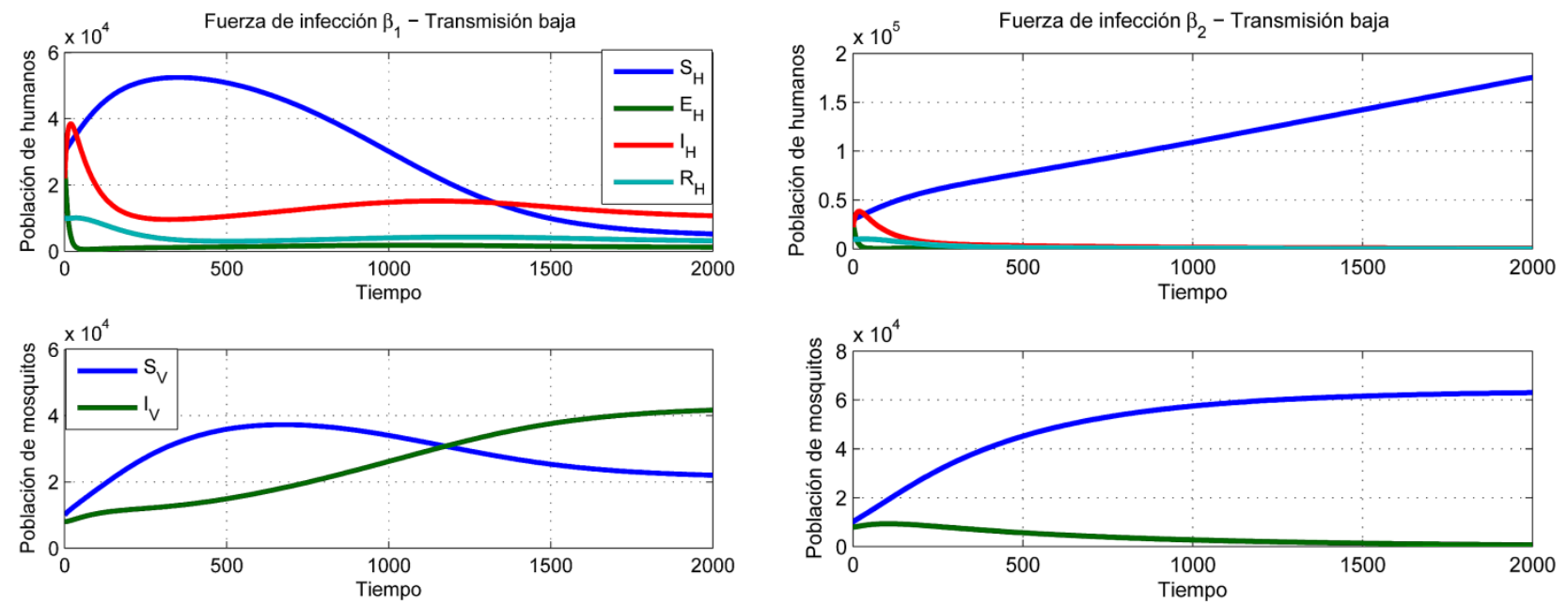

Figura 2. Gráficas para las simulaciones numéricas del sistema (6) en zonas de transmisión baja de SAT. A la izquierda considerando la fuerza de infección para mosquitos $\beta_{1}$ y a la derecha considerando $\beta_{2}$. En este escenario con $\beta_{1}$ se tiene que $R_{0}=0.56$ y las soluciones tienden al equilibrio $E=(5.230,1.242,10.720,3.171,22.040,41.660)$. Paran $\beta_{2}$ se tiene que $P_{0}=0.42$ y las soluciones tienden al equilibrio trivial. Las condiciones iniciales fueron $(30.000,28.000,22.000,10.000,10.000,8.00)$. Las simulaciones fueron realizadas usando ode 45 de MATLAB.

Por otro lado, el $R_{0}$ definido en (36) y el $P_{0}$ definido en (55) son independientes de la tasa de recuperación, además son crecientes con respecto a los parámetros $\beta_{h}$ y $\beta_{v}$, mientras que decrecen con respecto a los parámetros $\mu_{v}, \lambda$ y $\rho^{*}$, lo cual sugiere que para efectos de incorporar estrategias de control, se deberían tener en cuenta aquellas que afecten directamente la tasa de contacto entre humanos y mosquitos, tales como repelentes o toldillos, entre otros.

El análisis de existencia de soluciones de equilibrio mostró que la existencia de soluciones endémicas depende de la fuerza de infección considerada. Para el caso constante se evidencia la existencia de un solo equilibrio endémico el cual es local asintóticamente estable, sugiriendo la estabilidad global del equilibrio endémico. Para el caso no constante, bajo ciertas condiciones de los parámetros, existen uno o dos equilibrios endémicos, es decir, se sugiere la existencia de una región de bi-estabilidad, así como también de bifurcaciones transcríticas hacia adelante o hacia atrás.

Las simulaciones numéricas mostraron la posibilidad de disminuir los esfuerzos para controlar la enfermedad si se considera la fuerza de infección para los mosquitos $\beta_{2}$, lo cual sugiere que la enfermedad es más fácil de controlar cuando alguna de las fuerzas de infección es constante; es decir, cuando existe una relación de proporcionalidad entre la población total de individuos y la población de individuos infectados.

Para trabajos futuros se espera probar la estabilidad global del equilibrio endémico para el caso de fuerzas de infección constantes, así como también probar la existencia de bifurcaciones transcríticas. También se espera incluir parámetros de control para la enfermedad y hacer análisis costo-efectividad de las estrategias de control consideradas.

\section{AGRADECIMIENTOS}

Los autores agradecen a los evaluadores anónimos por sus sugerencias que ayudaron a mejorar el manuscrito. E. lbargüen-Mondragón agradece el apoyo recibido del proyecto No 083-20/11/2014 (VIPRI-UDENAR). Este trabajo está dedicado a la memoria del Dr. Anthony Afuwape quien en vida nos apoyó de manera incondional, QEPD.

\section{REFERENCIAS}

- Arino J, Ducrot A y Zongo P. 2012. A metapopulation model for malaria with transmissionblocking partial immunity in hosts. Journal of mathematical biology, 64(3), 423-448.

- Auger P, Kouokam E, Sallet G, Tchuente, M y Tsanou B. 2008. The RossMacdonald model in a patchy environment. Mathematical biosciences, 216(2), 123-131.

- Basáñez Gloria-María y Rodríguez Diego. 2004. Dinámica de transmisión y modelos matemáticos en enfermedades transmitidas por vectores. Entomotropica, 19(3), 113-134.

- Beaumont M, Zhang W y Balding D. 2002. Approximate Bayesian computation in population genetics. Genetics, 162(4), 2025-2035.

- Chitnis N. 2005. Using mathematical models in controlling the spread of malaria. In Partial Fulfillment of the Requirements For the Degree of Doctor OF Philosophy In the Graduate College. University of Arizona.

- Chitnis N, Cushing M y Hyman J. 2006. Bifurcation analysis of a mathematical model for malaria transmission. Society for Industrial and Applied Mathematics. 67(1),24-45.

- Chitnis N, Hyman J y Cushing M. 2008. Determining Important Parameters in the Spread of Malaria Through the Sensitivity Analysis of a Mathematical Model. Bulletin of Mathematical Biology, 70(5), 1272-1296.

- Cosner C, Beier J, Cantrell R, Impoinvil D, Kapitanski L, Potts M, Troyo A y Ruan S. 2009. The effects of human movement on the persistence of vectorborne diseases. Journal of theoretical biology, 258(4), 550-560. 
- Die C y Hasibeder G. 1986. Population dynamics of mosquito-borne disease: effects of flies which bite some people more frequently than others. Trans. R. Soc. Trop. Med. Hyg, 80 (1), 69-77.

- Dietz K, Molineaux L, Thomas A. 1974. A malaria model tested in the African savannah. Bull World Health Organ, 50(3-4), 347-357.

- Driessche P y Watmough J. 2002. Reproduction numbers and sub-threshold endemic equilibria for compartmental models of disease transmission. Math. Biosciences, 180, 29-48.

- Esteva L y Yang H. 2005. Mathematical model to assess the control of Aedes aegypti mosquitoes by the sterile insect technique. Mathematical biosciences, 198(2), 132-147.

- Ibargüen-Mondragón E, Esteva L y Chávez-Galán L. 2011. A mathematical model for cellular immunology of tuberculosis. J. Mathematical Eiosciences and Engineering, 8(4), 976-986.

- Fonseca J y Maestre A. 2009. Incidencia de las malarias gestacional, congénita y placentaria en Urabá (Antioquia, Colombia), 2005-2007. Revista colombiana de obstetricia y ginecología, 6o(1), 19-33.

- Gao D y Ruan S. 2012. A multipatch malaria model with logistic growth populations. SIAM journal on applied mathematics, 72(3), 819-841.

- Hasibeder G y Dye C. 1998. Population dynamics of mosquito-borne disease: persistence in a completely heterogeneous environments. Theoretical population biology, 33(1), 31-53.

- Macdonald G. 1957. The epidemiology and control of malaria. Oxford University, Press, London.

- Mandal S, Sarkar R y Sinha S. 2011. Mathematical models of malaria-a review. Malaria Journal, 10(1), 1.

- Mueller I, Schoepflin S, Smith TA, Benton KL, Bretscher MT, Lin E, Kiniboro B, Zimmerman PA, Speed TP, Siba P y Felger I. Force of infection is key to understanding the epidemiology of Plasmodium falciparum malaria in Papua New Guinean children. Proceedings of the National Academy of Sciences, 109(25), 10030-10035.
- Muench H. 1934. Derivation of rates from summation data by the catalytic curve. Journal of the American statistical association, 29(185), 25-38.

- Ngwa G and Shu W. 2000. A Mathematical Model for Endemic Malaria with Variable Human and Mosquito Populations. Mathematical and Computer Modeling, 32 (7), 747-763.

- Perko L. 1991. Differential Equations and Dynamical Systems. Springer Verlag, New York, First Edition.

- Rainey J, Mwanda W. O, Wairiumu P, Moormann A, Wilson M y Rochford R. 2007. Spatial distribution of Burkitt's lymphoma in Kenya and association with malaria risk. Tropical Medicine and International Health, 12(8), 936-943.

- Rodríguez D y Torres-Sorando L. 2001. Models of infectious diseases in spatially heterogeneous environments. Bulletin of Mathematical Biology, 63(3), 547-571.

- Roll Back malaria. The global partnership for a malaria-free world. Disponible en http://www.rbm.who.int/ProgressImpactSeries/report2.html. Consultado en enero 2014.

- Ross R. 1911. The prevention of malaria. John Murray, London.

- Smith D, Dushoff J y McKenzie F. 2004. The risk of a mosquito-borne infection in a heterogeneous environment. PLoS Biol, 2(11), 1957-1964.

- Sobrebón J, Cocho G, Aldama A, Falconi M, Esteva L y Lara M. 2002. Clásicos de la Biología Matemática. Siglo XXI editores, s.a de c.v. Cerro del agua 248 Delegación Coyoacán, 04310, México D.F. Primera edición.

- Tchuenche J, Chiyaka C, Chan D y Mayer G. A mathematical model for antimalarial drug resistance. 2011. Mathematical Medicine and Biology, 28 (24), 335-355.

- Torres-Sorando, L y Rodríguez D. 1997. Models of spatio-temporal dynamics in malaria. Ecological Modelling, 104(2), 231-240. 